\title{
Intelligent control of horizontal vibration of high-speed elevator based on gas-liquid active guide shoes
}

\author{
Qin $\mathrm{He}^{*}$, Peng Zhang, Shunxin Cao, Ruijun Zhang, and Qing Zhang \\ Department of Electromechanical Engineering, Shandong Jianzhu University, 1000 Feng Ming Road, Lingang Development Zone, \\ Jinan 250101, PR China
}

Received: 26 December 2019 / Accepted: 28 December 2020

\begin{abstract}
Aiming at the inconsistency between the vibration of the car and the car frame in the actual operation of a high-speed elevator and the horizontal vibration caused by the roughness excitation of the guide rail, this study designs a gas-liquid active guide shoe and establishes a horizontal vibration model of the 8-DOF high-speed elevator car system separated from the car and the car frame. Then, the correctness of the model is verified by experiments. Based on this, a fuzzy neural network intelligent vibration reduction controller based on the Mamdani model is designed and simulated by MATLAB. The results show that the root mean square value, mean value, and maximum value of vibration acceleration are reduced by more than $55 \%$ after using the fuzzy neural network control method, and the suppression effect is better than that of BP neural network control. Therefore, the intelligent vibration absorption controller designed by this research institute can effectively suppress the horizontal vibration of high-speed elevators.
\end{abstract}

Keywords: High-speed elevator / horizontal vibration / gas-liquid active guide shoe / fuzzy neural network / intelligent vibration reduction

\section{Introduction}

Nowadays, the number of high-rise and super-high-rise buildings is increasing. Furthermore, elevators are getting faster and faster. Increasing the speed of elevator operation brings about serious vibration problems. Research $[1,2]$ shows that in the actual operation of a high-speed elevator (with a lifting speed greater than $2 \mathrm{~m} / \mathrm{s}$ ), with the increase in elevator speed, the horizontal vibration problem of the elevator becomes more obvious. Higher horizontal vibration will cause passengers to experience discomfort and physiological fatigue. At the same time, the increase in horizontal vibration also affects the service life of elevator parts and reduces the safety of passengers riding in elevators. Therefore, how to effectively suppress the horizontal vibration of the car has become one of the key issues in the field of high-speed elevator research.

The literature shows that when the elevator's running speed is below $5 \mathrm{~m} / \mathrm{s}$, the main contributor to elevator horizontal vibration is the guide rail roughness excitation $[3,4]$. Therefore, as a direct contact with the guide rail in the elevator guidance system, guide shoes have become an

\footnotetext{
* e-mail: heqin67271@163.com
}

important research object in the vibration reduction work regarding high-speed elevators. Guide shoes are divided into passive guide shoes and active guide shoes. Passive guide shoes cannot meet the vibration damping demand of the high-speed elevator since it adapts only to the external disturbance input, which is not changed greatly, and thus, is unable to provide a large damping force. As the representative of active guide shoes, hydraulic guide shoes have become a new scheme to suppress the horizontal vibration of high-speed elevators because of their advantages of stable output, high control accuracy, and large control force [5-7].

In a high-speed elevator, the car and the car frame are connected by rubber blocks, which belong to the elastic connection mode. At the same time, since the car frame is not a regular cubic structure, the car and the car frame have centroid position deviation. Therefore, during the actual operation of the high-speed elevator, under the roughness excitation of the guide rail, the car and the car frame will exhibit an inconsistent vibration response. However, due to the lack of sufficient attention to this phenomenon, people only think that the stiffness of the car and the car frame are large, and that the deformation is small during operation. Therefore, many scholars regard the connection between car and car frame as rigid 
connection, when establishing a horizontal vibration model of the high-speed elevator to study the active control of the horizontal vibration of the high-speed elevator. Feng et al. [8-11] based on the theory of rigid body dynamics, simplified the guide shoe into a spring mass damping system, and simplified the car and the car frame into a rigid body. Then, an active control model for the horizontal vibration of the high-speed elevator with a guide shoe system was established. Next, based on the principle of hydraulic control, an elevator hydraulic active guide shoe was designed. Considering the similarity between the transverse vibration and lateral vibration of the car system, the transverse vibration of the elevator was taken as the research object, and a mathematical model of the elevator car with hydraulic active guide shoes was established. Based on the principle of constant pressure control and the fuzzy control algorithm, a fuzzy controller was then designed to suppress the horizontal vibration of the elevator. Finally, simulation results showed the feasibility of the designed hydraulic active guide shoe. Wang et al. $[12,13]$ made improved the hydraulic active guide shoes by adding a damping spring to increase the safety of the active guide shoes in case of control failure. An electro-hydraulic servo valve and two pressure sensors were used to control the two chambers of the hydraulic actuator at constant pressure. Meanwhile, an acceleration sensor was installed on the base seat of the guide shoe, which formed a double feedback control of pressure and acceleration, and improved the control response speed and accuracy. Based on this, a dynamic model of 6-DOF active control of horizontal vibration of the high-speed elevator (with the car and the car frame integrated) was established. The adaptive fuzzy control algorithm was used to design the controller to suppress the horizontal vibration of the elevator. Finally, experimental verification of the Aduc 7026-based high-speed elevator hydraulic active guide shoe control system was carried out, and the results proved the effectiveness of the designed guide shoe. Although the above scholars restrained the horizontal vibration of highspeed elevators to a certain extent, the difference between the vibration response of the car and the car frame in the actual operation of high-speed elevators was neglected. They regard the car and the car frame as a rigid body for analysis, that is, the vibration acceleration of the car is represented by the vibration acceleration of the car frame, which is obviously unreasonable. The accuracy of this elevator model is low, and the research on active vibration control based on this model will affect the control accuracy. At the same time, due to the shortcomings of the hydraulic control system itself, such as oil leakage, slow response speed and oil has no buffering effect, which lead to some problems with the hydraulic active guide shoes designed, such as slow control response, oil leakage and ride comfort caused by lack of buffering effect. Therefore, the hydraulic active guide shoes designed have poor suppression effect on the horizontal vibration of high-speed elevator.

In terms of control algorithms, Nakano et al. [14] used the acceleration response of the car system as the control objective and applied the optimal feedback control method to control the horizontal vibration acceleration of the car, thus achieving suppression of the horizontal vibration of the car system. Naoaki et al. [15] used the $\mathrm{H}_{\infty}$ control method to design a robust controller for the horizontal vibration of the car system. Xue et al. [16] adopted the strategy of combining generalized predictive control with PID and designed a generalized predictive PID controller to suppress horizontal vibration. Santo et al. [17] proposed a control strategy based on the state-dependent Riccati equation (SDRE) to achieve elevator vibration reduction. The control algorithms applied in the above studies did not have a self-learning ability for the random excitation of the car system, and the control effect was slightly poor. Furthermore, Zhang et al. [18] designed a PID controller based on the BP neural network to control the horizontal vibration of high-speed elevators. Although the control algorithm could perform self-learning, memory, and other intelligent processing on random excitation, the algorithm required a large amount of data for network training to ensure the accuracy of control. Meanwhile, due to the black box nature of the algorithm, it cannot give a clear reasoning basis for its output results, and the algorithm cannot apply the existing expert knowledge and experience.

Aimed at the problems of slow control response, oil leakage, and no buffering effect in the active control research of hydraulic shoes, this study designs a gas-liquid active guide shoe using a gas-liquid converter and a gasliquid cylinder. Considering the non-coincidence of the centroid of the car and the car frame in the actual structure of the high-speed elevator and the difference in vibration response between the car and the car frame during actual operation, and applying the designed active guide shoes to the high-speed elevator, a horizontal vibration model of the 8-DOF high-speed elevator with active control separated from the car and the car frame is established. Then the correctness of the model is verified by experiments. Combining fuzzy control with neural network control, a fuzzy neural network intelligent controller based on the Mamdani model is designed. Finally, simulation analysis is carried out in MATLAB to verify the effectiveness of the designed controller.

\section{Structure and control principle of gas-liquid active guide shoe}

In view of the shortcomings of the existing hydraulic active guide shoes, such as oil leakage, slow response speed, and no buffering effect, this study uses pneumatic transmission as a power source because of its advantages of quick action, low cost, and overload protection. Meanwhile, considering the problems of low control accuracy and poor stability of pneumatic transmission, the combination of a gas-liquid converter and a gas-liquid cylinder is used to improve the pneumatic transmission in order to increase its transmission accuracy and stability. A gas-liquid active guide shoe based on the gas-liquid converter is designed and applied to the high-speed elevator. Then the active vibration reduction of the car system is realized through the design of an intelligent controller. 


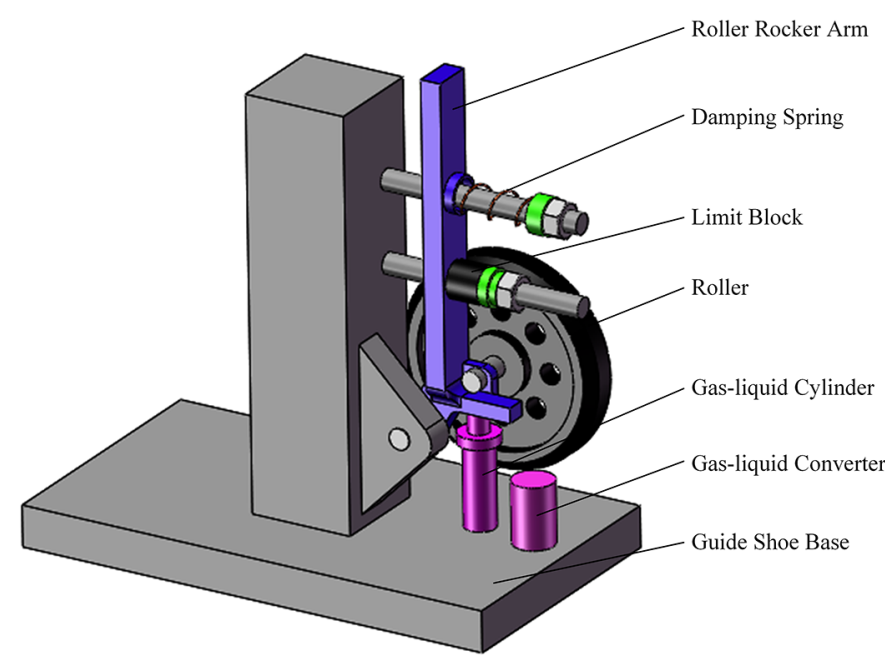

Fig. 1. Schematic of the gas-liquid active guide shoe.

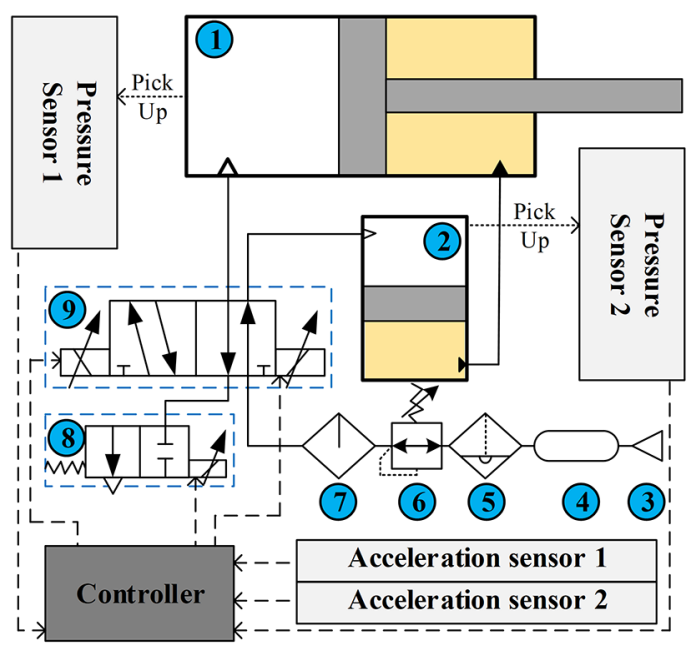

1. Gas-liquid cylinder 2. Gas-liquid converter 3. Gas source 4. Gas storage tank 5. Filter 6. Pressure reducing valve 7. Atomized lubricator

8. Proportional electric reversing valve 9. Proportional electric reversing valve

Fig. 2. Principle diagram of gas-liquid active guide shoe.

The schematic of the designed gas-liquid active guide shoe is shown in Figure 1. It consists of a guide shoe base, roller rocker arm, roller, damping spring, limit block, gasliquid cylinder, gas-liquid converter, pressure sensor, acceleration sensor, controller, pneumatic source, gas storage tank, three pneumatic parts (FRL: filter, pressure reducing valve, atomized lubricator), proportional electric reversing valve and so on. The push rod of the gas-liquid cylinder is hinged with the rocker arm of the roller, and the other end of the gas-liquid cylinder is hinged with the base of the guide shoe.

The control system adopts the control principle combining acceleration error feedback control and pressure error compensation control, as shown in Figure 2. When the elevator is running, acceleration vibration sensor 1 and acceleration sensor 2, respectively, detect the horizontal vibration accelerations $a_{1}$ and $a_{2}$ of the car and the car frame. Then the errors $\Delta a 1$ and $\Delta a 2$ between the signal and the expected horizontal vibration acceleration are fed into the controller for control calculation. The control current $i$ is obtained, and the opening direction and size of the proportional electric reversing valve are controlled by the current $i$; thus, the theoretical flow of gas is controlled. The theoretical control force $u_{1}$ is obtained. Considering the problem of inaccurate control force due to the compressibility of the gas, pressure sensors 1 and 2 are used to detect pressures $p_{1}$ and $p_{2}$, respectively, of the pneumatic cylinder of the gas-liquid cylinder and the gas-liquid converter. The sensors also send the pressure error $\Delta p$ feedback to the controller for compensation control, thereby correcting the control force $u_{1}$, and obtaining an accurate control force $u$. Then, the roller rocker arm is pushed by the expansion and contraction of the gas-liquid cylinder to achieve precise control of the horizontal vibration of the car system.

\section{Horizontal vibration dynamics model and its control problem}

\subsection{Establishment of a horizontal vibration dynamics model of a high-speed elevator car system with active control}

This study only considers the horizontal vibration between the elevator car system (collectively referred to as the car system) and the guide rail, that is, the vibration parallel to the direction of the car door (including the translation of the car system and the rotation around its center of mass). In order to simplify the model, the following reasonable assumptions are made according to the elevator structure and its motion law [19]:

- Considering the different sizes of the car and the car frame in the actual elevator, and the connection between the car and the car frame through rubber blocks, the car and the car frame are separated and modeled. The rubber blocks between the car and the car frame are simplified as a spring damping system. Therefore, the car system can be simplified as a mass spring damping system.

- Since the roller is in close contact with the surface of the guide rail under the preloading force, the relative displacement between them is small in actual operation; therefore, the connection between the guide shoe roller and the guide rail can be simplified as a mass spring damping system.

- The connection between the car system and the guide shoe through the damping spring and the rubber limit block, and the relative displacement, is small; thus, the connection between the car system and the guide shoe is simplified as a mass spring damping system.

- The structure and parameters of each roller are identical.

- We only consider the guide rail roughness excitation.

On the basis of the above assumptions and the model in the reference [19], we consider the gas-liquid active guide shoe designed in Section 1 and establish the horizontal vibration model of the 8-DOF high-speed elevator with 
active control (Fig. 3). In Figure 3, $O_{c}$ and $O_{f}$ are the centroid of the car and the car frame, respectively. The rotation of the car about its centroid $O_{c}$ is $\theta_{c}$, and the rotation of the car frame about its centroid $O_{f}$ is $\theta_{f}$. The mass of the car is $m_{c}$, and the moment of inertia is $J_{c}$. The mass of the car frame is $m_{f}$, and the moment of inertia is $J_{f}$. The horizontal displacement of the car's centroid is $x_{c}$, the horizontal displacement of the centroid of the car frame is $x_{f}$, and the horizontal displacement of the guide roller is $x_{s i}(i=1,2,3,4)$. The stiffness and damping coefficients between the roller and the guide rail are $k_{r}$ and $c_{r}$. The stiffness and damping coefficients between the roller and the car system are $k_{s}$ and $c_{s}$. The stiffness and damping coefficients of the rubber block are $k_{a}$ and $c_{a}$. The vertical distances from rubber block 1, 2 to the centroid of the car is $l_{1}$. The vertical distances from rubber block 3,4 to the centroid of the car is $l_{2}$. The vertical distances from rubber block 1,2 to the centroid of the car frame is $l_{5}$. The vertical distances from rubber block 3, 4 to the centroid of the car frame is $l_{6}$. The vertical distances from guide shoe 1,2 to the centroid of the car frame is $l_{3}$. The vertical distances from guide shoe 3,4 to the centroid of the car frame is $l_{4}$. The roughness excitation of the guide rail (displacement form) is $x_{r i}(i=1,2,3,4)$, and the active control force is $f_{i}$ $(i=1,2,3,4)$.

The dynamic equation of the model is obtained by the Lagrange energy method:

where

$$
\text { See equation (1) below. }
$$

\section{See equation below.}

\subsection{Control problem description}

Since the horizontal vibration of the high-speed elevator is aggravated by the roughness excitation of the guide rail, this study designs a gas-liquid active guide shoe to suppress it. The evaluation index of the horizontal vibration of the high-speed elevator is the horizontal vibration acceleration of the car system [20,21]. Therefore, the control objective of this study is to set the horizontal vibration acceleration of the car system.

$$
\begin{aligned}
& \left\{\begin{array}{l}
M_{1} \ddot{q}+C_{1} \dot{q}+C_{2} \dot{x}_{s}+K q+K_{1} x_{s}=G F \\
M_{r} \ddot{x}_{s}+C_{2}^{T} \dot{q}+\left(C_{s}+C_{r}\right) \dot{x}_{s}+G_{1} C_{r} \dot{x}_{r}+K_{1}^{T} q+\left(K_{s}+K_{r}\right) x_{s}+G_{1} K_{r} x_{r}=G_{1} F
\end{array}\right. \\
& q=\left[\begin{array}{llll}
x_{c} & x_{f} & \theta_{c} & \theta_{f}
\end{array}\right]^{T}, x_{s}=\left[\begin{array}{llll}
x_{s 1} & x_{s 2} & x_{s 3} & x_{s 4}
\end{array}\right]^{\mathrm{T}}, x_{r}=\left[\begin{array}{llll}
x_{r 1} & x_{r 2} & x_{r 3} & x_{r 4}
\end{array}\right]^{\mathrm{T}}, \\
& C_{1}=\left[\begin{array}{ccll}
4 c_{a} & -4 c_{a} & 2 c_{a}\left(l_{2}-l_{1}\right) & 2 c_{a}\left(l_{5}-l_{6}\right) \\
-4 c_{a} & 4 c_{a}+4 c_{s} & 2 c_{a}\left(l_{1}-l_{2}\right) & 2 c_{a}\left(l_{6}-l_{5}\right)+2 c_{s}\left(l_{4}-l_{3}\right) \\
2 c_{a}\left(l_{2}-l_{1}\right) & 2 c_{a}\left(l_{1}-l_{2}\right) & 2 c_{a}\left(l_{1}^{2}+l_{2}^{2}\right) & -2 c_{a}\left(l_{1} l_{5}+l_{2} l_{6}\right) \\
2 c_{a}\left(l_{5}-l_{6}\right) & 2 c_{a}\left(l_{6}-l_{5}\right)+2 c_{s}\left(l_{4}-l_{3}\right) & -2 c_{a}\left(l_{1} l_{5}+l_{2} l_{6}\right) & 2 c_{a}\left(l_{5}^{2}+l_{6}^{2}\right)+2 c_{s}\left(l_{3}^{2}+l_{4}^{2}\right)
\end{array}\right] \\
& K=\left[\begin{array}{cccl}
4 k_{a} & -4 k_{a} & 2 k_{a}\left(l_{2}-l_{1}\right) & 2 k_{a}\left(l_{5}-l_{6}\right) \\
-4 k_{a} & 4 k_{a}+4 k_{s} & 2 k_{a}\left(l_{1}-l_{2}\right) & 2 k_{a}\left(l_{6}-l_{5}\right)+2 k_{s}\left(l_{4}-l_{3}\right) \\
2 k_{a}\left(l_{2}-l_{1}\right) & 2 k_{a}\left(l_{1}-l_{2}\right) & 2 k_{a}\left(l_{1}^{2}+l_{2}^{2}\right) & -2 k_{a}\left(l_{1} l_{5}+l_{2} l_{6}\right) \\
2 k_{a}\left(l_{5}-l_{6}\right) & 2 k_{a}\left(l_{6}-l_{5}\right)+2 k_{s}\left(l_{4}-l_{3}\right) & -2 k_{a}\left(l_{1} l_{5}+l_{2} l_{6}\right) & 2 k_{a}\left(l_{5}^{2}+l_{6}^{2}\right)+2 k_{s}\left(l_{3}^{2}+l_{4}^{2}\right)
\end{array}\right] \\
& K_{1}=\left[\begin{array}{cccl}
0 & 0 & 0 & 0 \\
-k_{s} & -k_{s} & -k_{s} & -k_{s} \\
0 & 0 & 0 & 0 \\
k_{s} l_{3} & k_{s} l_{3} & -k_{s} l_{4} & -k_{s} l_{4}
\end{array}\right], C_{2}=\left[\begin{array}{cccl}
0 & 0 & 0 & 0 \\
-c_{s} & -c_{s} & -c_{s} & -c_{s} \\
0 & 0 & 0 & 0 \\
c_{s} l_{3} & c_{s} l_{3} & -c_{s} l_{4} & -c_{s} l_{4}
\end{array}\right], G=\left[\begin{array}{cccc}
0 & 0 & 0 & 0 \\
1 & 1 & 1 & 1 \\
0 & 0 & 0 & 0 \\
-l_{3} & l_{3} & l_{4} & -l_{4}
\end{array}\right] \\
& G_{1}=\operatorname{diag}(-1,-1,-1,-1), M_{r}=\operatorname{diag}\left(m_{r}, m_{r}, m_{r}, m_{r}\right), C_{s}=\operatorname{diag}\left(c_{s}, c_{s}, c_{s}, c_{s}\right) \text {, } \\
& C_{r}=\operatorname{diag}\left(c_{r}, c_{r}, c_{r}, c_{r}\right), M_{1}=\operatorname{diag}\left(m_{c}, m_{f}, J_{c}, J_{f}\right), K_{s}=\operatorname{diag}\left(k_{s}, k_{s}, k_{s}, k_{s}\right), \\
& K_{r}=\operatorname{diag}\left(k_{r}, k_{r}, k_{r}, k_{r}\right), F=\left[\begin{array}{llll}
f_{1} & f_{2} & f_{3} & f_{4}
\end{array}\right]^{T} .
\end{aligned}
$$




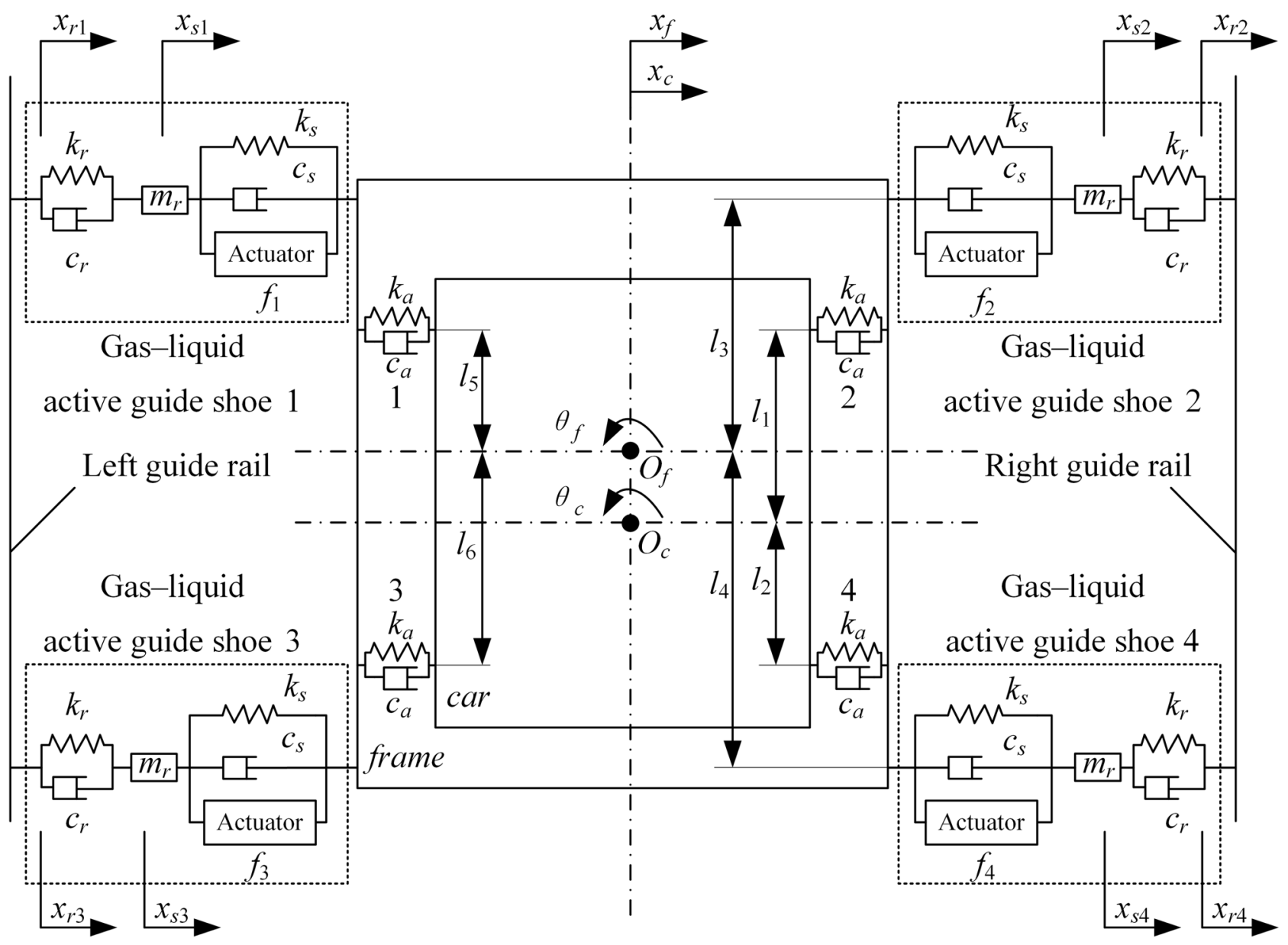

Fig. 3. Horizontal vibration model of the high-speed elevator car system with active control.

Considering that the horizontal vibration of the car system is a multi-input and multi-output control problem, the state space equation is used to describe equation (1) of the model as follows.

The state vector is selected as $X=\left[\begin{array}{llll}q & x_{s} & \dot{q} & \dot{x}_{s}\end{array}\right]^{T}$ $\in R^{16 \times 1}$, the control vector is $U=\left[\begin{array}{llll}f_{1} & f_{2} & f_{3} & f_{4}\end{array}\right]^{T} \in R^{4 \times 1}$, the interference input vector is $W=\left[\begin{array}{ll}x_{r} & \dot{x}_{r}\end{array}\right]^{T} \in R^{8 \times 1}$, and the output vector is $y=\left[\begin{array}{llll}\ddot{x}_{c} & \ddot{x}_{f} & \ddot{\theta}_{c} & \ddot{\theta}_{f}\end{array}\right]^{T} \in R^{4 \times 1}$. Then the state space equation of the system can be expressed as follows:

$$
\left\{\begin{array}{l}
\dot{X}=A X+B U+R W \\
y=C X+D U
\end{array}\right.
$$

where

See equation below.

$$
\begin{aligned}
& A=\left[\begin{array}{cccl}
0_{4} & 0_{4} & I_{4} & 0_{4} \\
0_{4} & 0_{4} & 0_{4} & I_{4} \\
-M_{1}^{-1} K & -M_{1}^{-1} K_{1} & -M_{1}^{-1} C_{1} & -M_{1}^{-1} C_{2} \\
-M_{r}^{-1} K_{1}^{T} & -M_{r}^{-1}\left(K_{s}+K_{r}\right) & -M_{r}^{-1} C_{2}^{T} & -M_{r}^{-1}\left(C_{s}+C_{r}\right)
\end{array}\right], B=\left[\begin{array}{l}
0_{8 \times 4} \\
M_{1}^{-1} G \\
M_{r}^{-1} G_{1}
\end{array}\right], \\
& C=\left[\begin{array}{llll}
-M_{1}^{-1} K & -M_{1}^{-1} K_{1} & -M_{1}^{-1} C_{1} & -M_{1}^{-1} C_{2}
\end{array}\right], R=\left[\begin{array}{cc}
0_{12 \times 4} & 0_{12 \times 4} \\
-M_{r}^{-1} G_{1} K_{r} & -M_{r}^{-1} G_{1} C_{r}
\end{array}\right], D=M_{1}^{-1} G .
\end{aligned}
$$




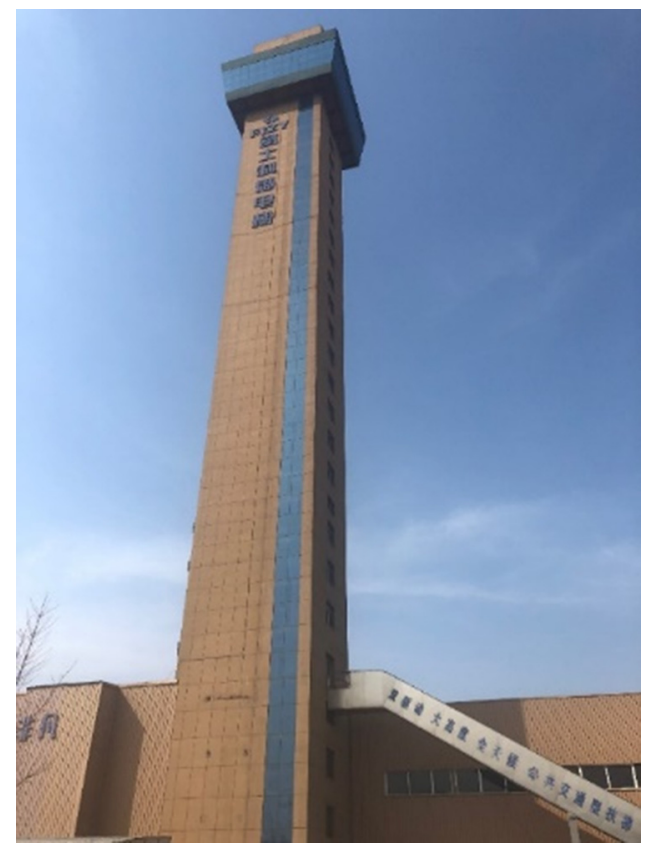

Fig. 4. The high-speed elevator test tower.

\section{Model verification}

To ensure the active control method of horizontal vibration is accurate, we shall verify the horizontal vibration model of high-speed elevators established in Section 2 is appropriate.

\subsection{Experimental measurement}

The test took place on November 23, 2020, in the highspeed elevator test tower (Fig. 4) of Shandong Fuji Zhiyu Elevator Co, Ltd. $4 \mathrm{~m} / \mathrm{s}$ high-speed elevator (Fig. 5) was used for the test and the test equipment was a DT-4A elevator acceleration tester (Fig. 6). The vibration acceleration measurement range of the equipment is -980 to $980 \mathrm{~cm} / \mathrm{s}^{2}$ in the vertical direction and -120 to $120 \mathrm{~cm} / \mathrm{s}^{2}$ in the horizontal direction; The sampling interval is $0.01 \mathrm{~s}$, and the maximum continuous data collection time is $2000 \mathrm{~s}$; The instrument size is $182 \mathrm{~mm} \times$ $104 \mathrm{~mm} \times 44 \mathrm{~mm}$.

As the evaluation standard for elevator riding comfort is the horizontal acceleration of the center of the car floor, the measuring points of the DT-4A elevator vibration acceleration tester are selected in the center of the car floor and the center of the bottom of the car frame during the test. The location of the measuring point is shown in Figures 7 and 8 .

\subsection{Numerical Simulation}

Utsunomiy et al. [22] conducted a study of guide rail excitation's energy is mainly concentrated in the lowfrequency power. According to [23-25], the guide rail's

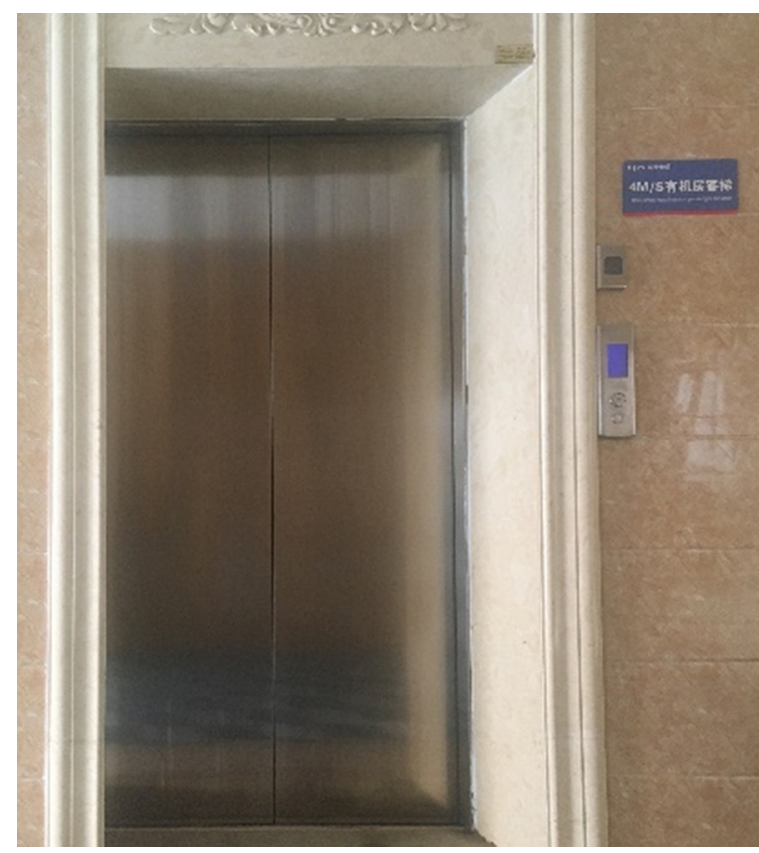

Fig. 5. $4 \mathrm{~m} / \mathrm{s}$ high-speed elevator.

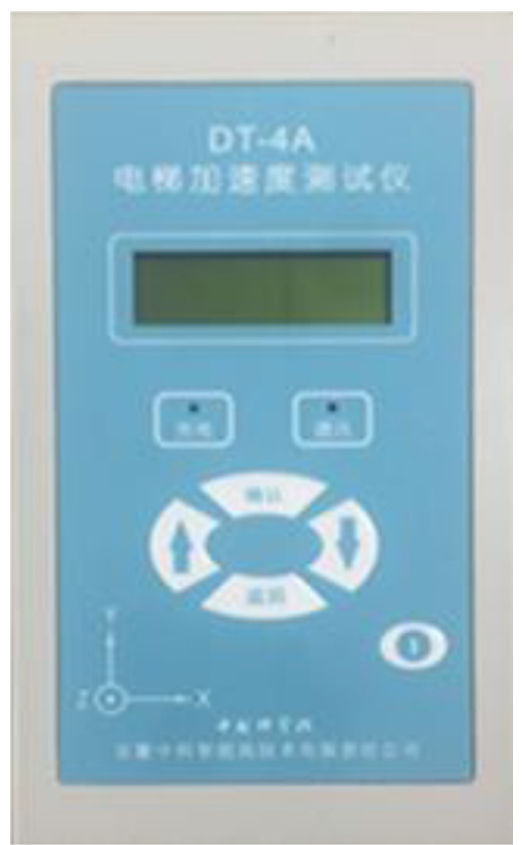

Fig. 6. DT-4A elevator acceleration tester.

surface roughness error approximately conforms to a normal distribution. The mean value is 0 , and the standard deviation is $0.6 \mathrm{~mm}$. Therefore, according to the statistical characteristics of guide rail roughness excitation, the guide roughness excitation, as shown in Figure 9, is generated.

In the simulation, the surface roughness excitation of the guide rail, as shown in Figure 7, was brought into equation (2) for the simulation solution, and the simulated 


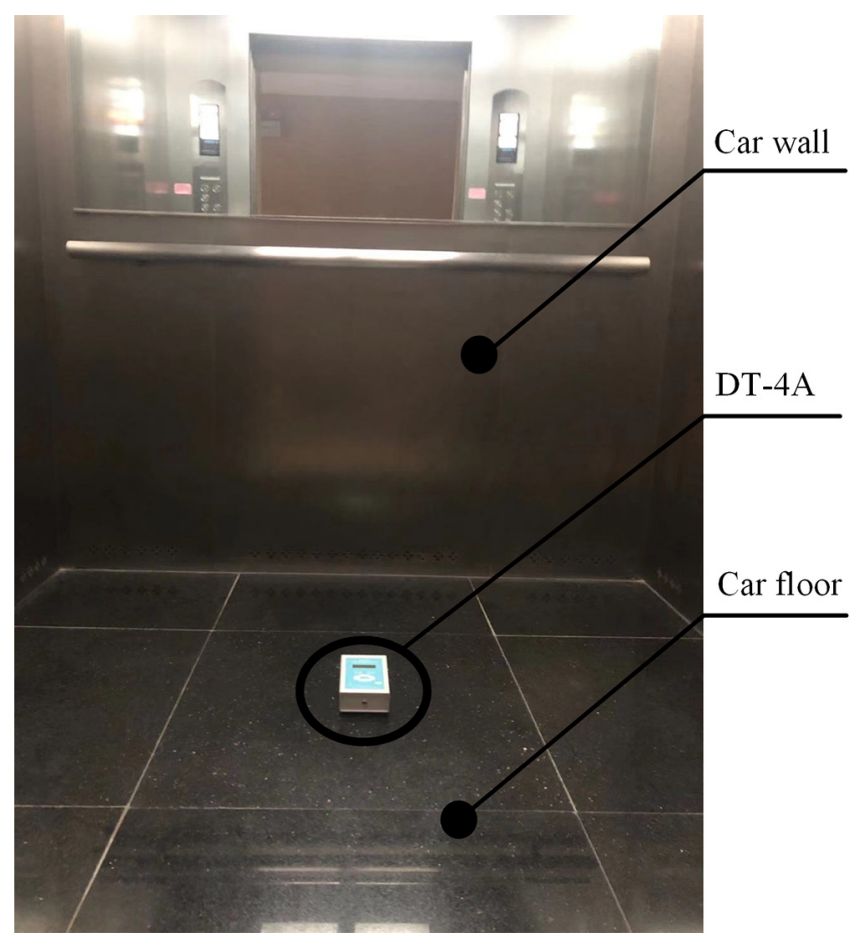

Fig. 7. The measuring points of the car.

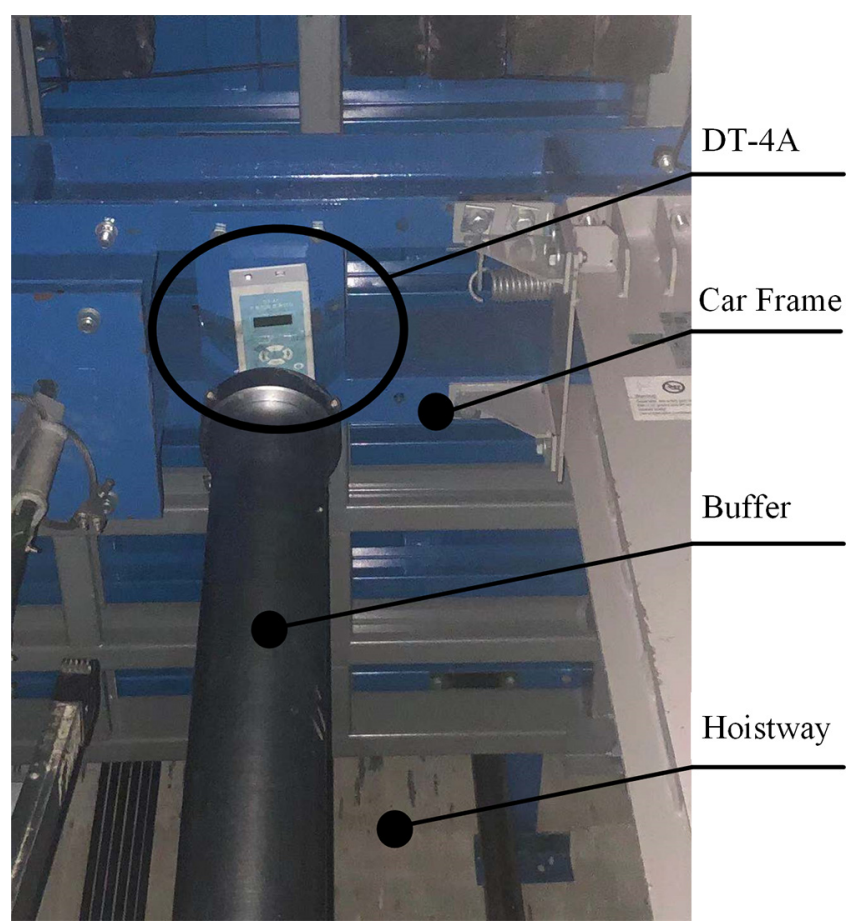

Fig. 8. The measuring points of the car frame.

horizontal vibration acceleration of the car and the car frame was obtained. The detailed parameters of the highspeed elevator are shown in Table 1.
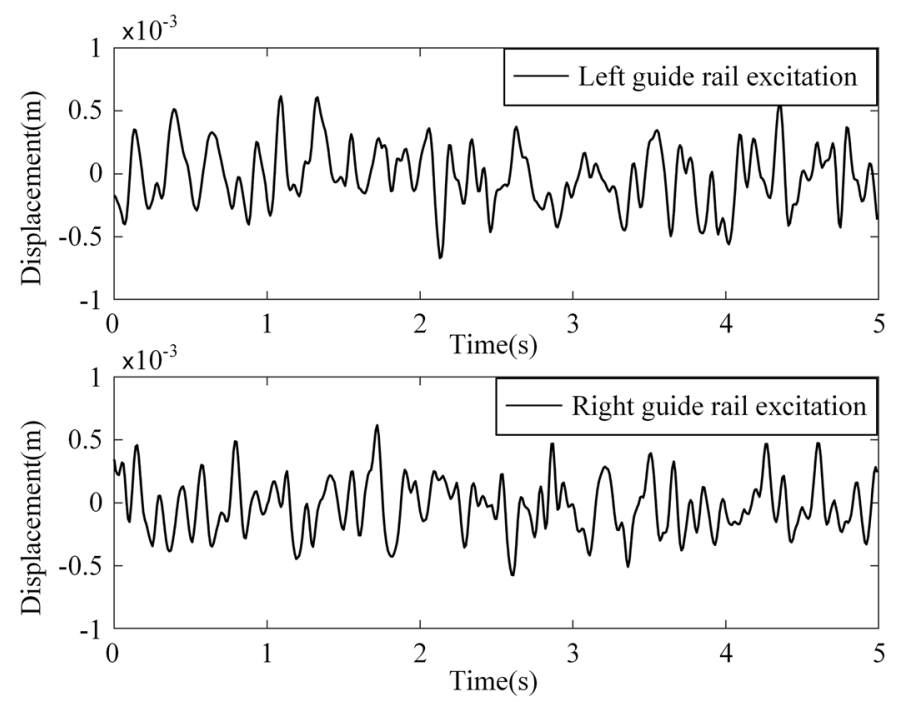

Fig. 9. Guide rail excitation.

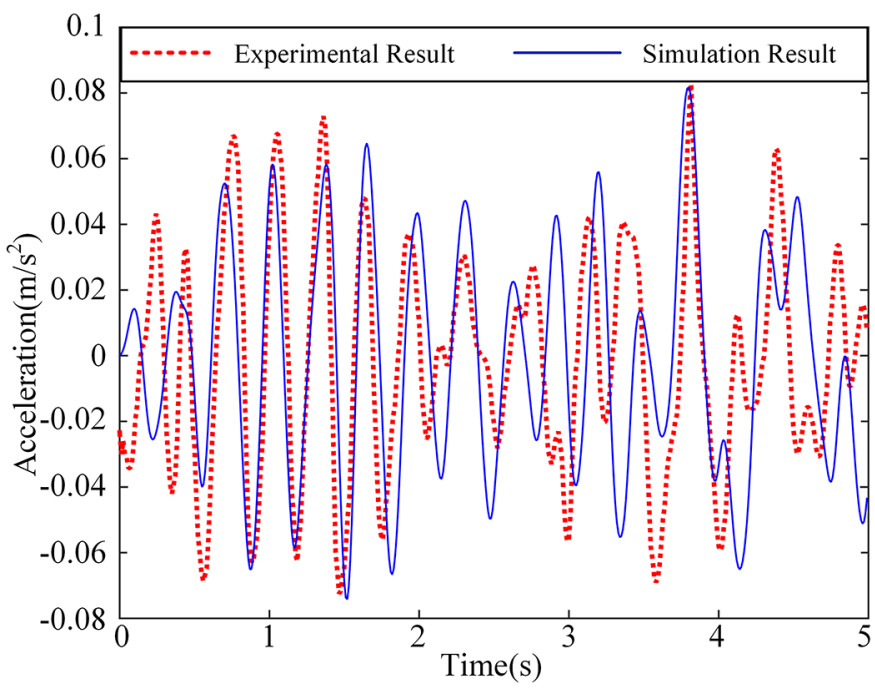

Fig. 10. Time-domain diagram contrasting the actual acceleration and the simulated acceleration of the car.

\subsection{Analysis of verification results}

The simulated horizontal vibration acceleration curves of the car in the time domain and frequency domain are obtained and compared with the measured curves, as shown in Figures 10 and 12. The simulated horizontal vibration acceleration curves of the car frame in the time domain and frequency domain are obtained and compared with the measured curves, as shown in Figures 11 and 13.

Figures 10 and 11 show, in the time domain that the simulation results of the horizontal vibration acceleration of the car and car frame are consistent with the trend of the response curve, which is measured when the high-speed elevator is disturbed by the uneven guide rail excitation. Figures 12 and 13 show, the simulated and test results of horizontal vibration acceleration of the car body and the car frame are generally concentrated in the low-frequency 
Table 1. High-speed elevator parameters.

\begin{tabular}{|c|c|c|c|}
\hline Parameter & $\begin{array}{l}\text { Numerical } \\
\text { value }\end{array}$ & Parameter & $\begin{array}{l}\text { Numerical } \\
\text { value }\end{array}$ \\
\hline Mass of car $m_{c}(\mathrm{~kg})$ & $1.2 \mathrm{e} 3$ & $\begin{array}{l}\text { Damping coefficient between guide shoe } \\
\text { and car frame } c_{s}(\mathrm{~N} \cdot \mathrm{s} / \mathrm{m})\end{array}$ & $1.2 \mathrm{e} 2$ \\
\hline Rated load $m_{\max }(\mathrm{kg})$ & $1.0 \mathrm{e} 3$ & $\begin{array}{l}\text { Stiffness coefficient of damping } \\
\text { block } k_{a}\left(\mathrm{~N} \cdot \mathrm{m}^{-1}\right)\end{array}$ & $5.0 \mathrm{e} 5$ \\
\hline Moment of inertia of car $J_{c}\left(\mathrm{~kg} \cdot \mathrm{m}^{2}\right)$ & $1.3 \mathrm{e} 3$ & $\begin{array}{l}\text { Damping coefficient of damping } \\
\text { block } c_{a}(\mathrm{~N} \cdot \mathrm{s} / \mathrm{m})\end{array}$ & $3.2 \mathrm{e} 2$ \\
\hline Mass of car frame $m_{f}(\mathrm{~kg})$ & $7.5 \mathrm{e} 2$ & Distance $l_{1}(\mathrm{~m})$ & 1.17 \\
\hline Moment of inertia of car frame $J_{f}\left(\mathrm{~kg} \cdot \mathrm{m}^{2}\right)$ & $3.0 \mathrm{e} 3$ & Distance $l_{2}(\mathrm{~m})$ & 1.18 \\
\hline Mass of roller $m_{r}(\mathrm{~kg})$ & 3.1 & Distance $l_{3}(\mathrm{~m})$ & 1.6 \\
\hline $\begin{array}{l}\text { Stiffness coefficient between } \\
\text { guide shoe and guide rail } k_{r}\left(\mathrm{~N} \cdot \mathrm{m}^{-1}\right)\end{array}$ & $7.0 \mathrm{e} 5$ & Distance $l_{4}(\mathrm{~m})$ & 1.4 \\
\hline
\end{tabular}

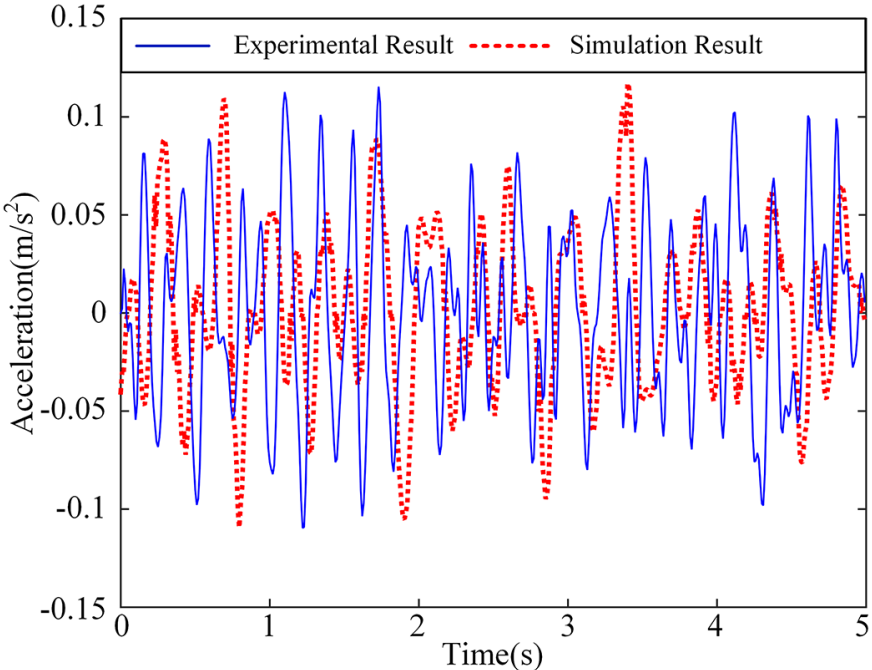

Fig. 11. Time-domain diagram contrasting the actual acceleration and the simulated acceleration of the car frame.

range of $0-5 \mathrm{~Hz}$, which is the same trend. So the simulation results are in high agreement with the test measurement results.

\subsubsection{Error analysis}

The comparison results of the root mean square value, mean value, and maximum value of acceleration are shown in Table 2.

According to $[26,27]$, the relative error calculation equation is:

$$
\Delta e=\frac{\left|x_{a}-x_{m}\right|}{x_{m}} \times 100 \%
$$

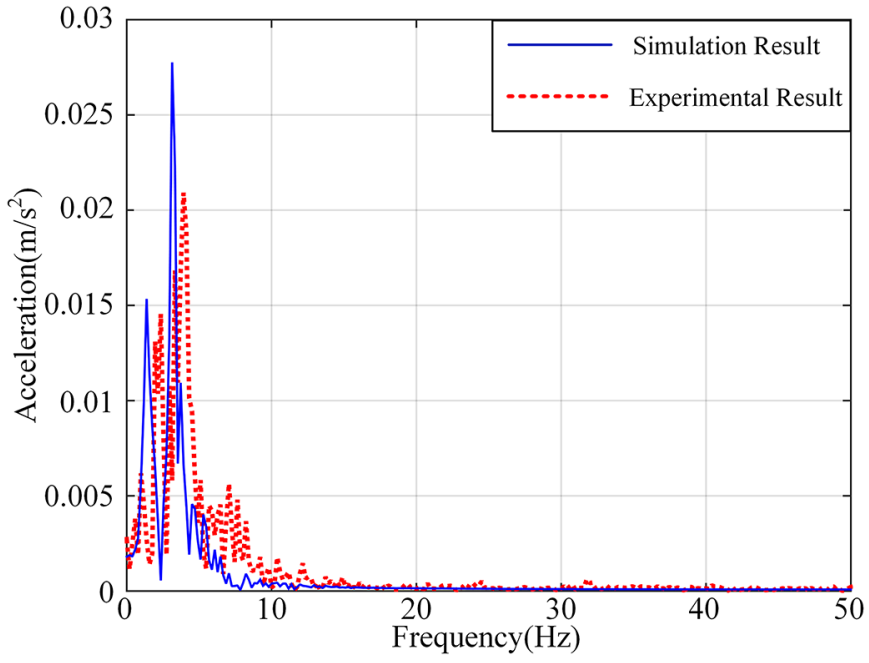

Fig. 12. Frequency-domain diagram contrasting the actual acceleration and the simulated acceleration of the car.

where, $\Delta_{e}$ is the relative error, $x_{a}$ is the simulation result, and $x_{m}$ is the test measurement result.

According to the comparison of the test measurement results and the simulation results in Table 2 and the equation (3), the relative errors of the average value, root mean square value and maximum value of the horizontal car vibration acceleration are $4.63 \%, 2.05 \%$ and $4.44 \%$ respectively; The relative errors of the mean, mean square and maximum acceleration of the car frame are $7.12 \%$, $4.62 \%$ and $1.54 \%$ respectively. Therefore, the solution results of the model established in this paper are in good agreement with the experimental measurement results, which proves the model established is accuracy.

There are several reasons for the errors showing in the data comparison: 
Table 2. Time-domain response results of horizontal vibration acceleration of the car and the car frame.

\begin{tabular}{lllll}
\hline & & RMS $\left(\mathrm{m} / \mathrm{s}^{2}\right)$ & MEAN $\left(\mathrm{m} / \mathrm{s}^{2}\right)$ & $\operatorname{MAX}\left(\mathrm{m} / \mathrm{s}^{2}\right)$ \\
\hline Experimental & Car & 0.0342 & 0.0281 & 0.0855 \\
Result & Car frame & 0.0455 & 0.0365 & 0.1172 \\
Simulation & Car & 0.0349 & 0.0294 & 0.0817 \\
Result & Car frame & 0.0476 & 0.0391 & 0.1154 \\
\hline
\end{tabular}

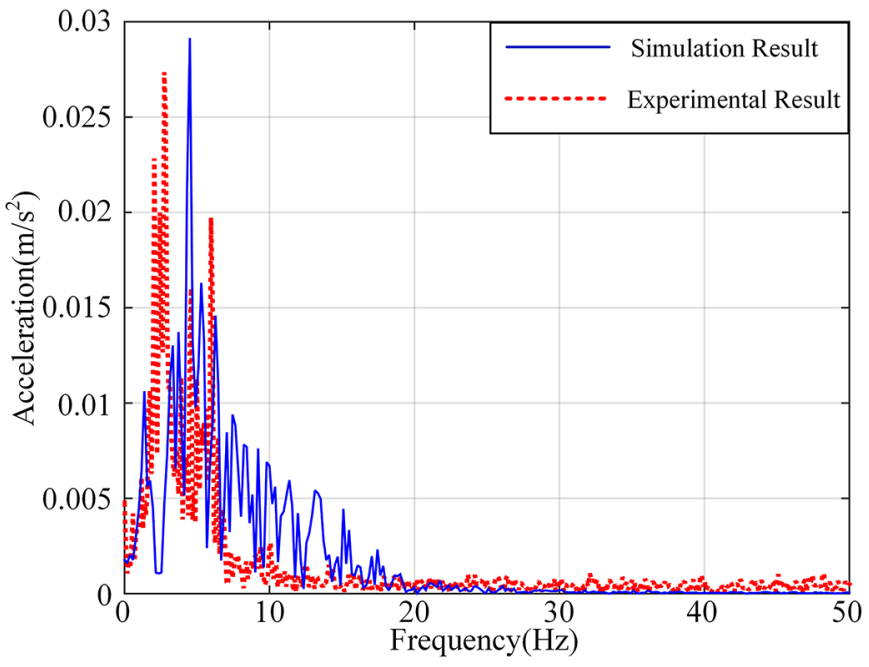

Fig. 13. Frequency-domain diagram contrasting the actual acceleration and the simulated acceleration of the car frame.

- There are errors in the dimensions, stiffness, damping, and other parameters between the car system model established in this paper and the actual car system, resulting in errors in the acceleration response between the established numerical model and the actual model.

- The test elevator's maximum actual running speed is slightly less than $4 \mathrm{~m} / \mathrm{s}$, and there are errors between it and the speed used in the simulation solution.

- The position between the DT-4A elevator acceleration tester and the measuring point is offset, resulting in errors.

- The surface roughness excitation of the guide rail generated in simulation is a statistical result, which has errors with the actual surface roughness excitation of the guide rail.

\subsubsection{Model goodness of fit}

The determination coefficient $R^{2}$ is used to test the accuracy of the model. Its calculation equation $[28,29]$ is:

$$
R^{2}=\frac{\sum_{i=1}^{n}\left(\hat{y}_{i}-\bar{y}_{i}\right)^{2}}{\sum_{i=1}^{n}\left(y_{i}-\bar{y}_{i}\right)^{2}}=1-\frac{\sum_{i=1}^{n}\left(y_{i}-\hat{y}_{i}\right)^{2}}{\sum_{i=1}^{n}\left(y_{i}-\bar{y}_{i}\right)^{2}}
$$

where, $y_{i}$ is the test measurement result, $\hat{y}_{i}$ is the simulation solution result, $\bar{y}_{i}$ is the average of the test measurement result. The closer the value of $R^{2}$ to 1 , the higher the accuracy of the model. When $R^{2}>0.4$, it indicates the fitting effect is good.

According to equation (4), the car's horizontal vibration acceleration model's determining coefficient can be calculated as 0.9650 , and the determining coefficient of the car frame horizontal vibration acceleration model is 0.8415 . Therefore, the model established in this paper has an excellent fitting degree, which further proves the correctness of the model established in this paper.

\section{Fuzzy neural network intelligent vibration absorption controller based on the Mamdani model}

Neural network control (NNC) [30-32] is a representative of modern intelligent control. It is an active network composed of a simple computing-processing unit (i.e., a neuron) and a network topology. Neural network control has good ability of learning, memory and fast computation. It also has good adaptability to uncertain factors such as the roughness excitation of the guide rail and changes in car load during actual operation of the high-speed elevator. Moreover, through the learning algorithm (error back propagation algorithm), the horizontal vibration of the car system can be suppressed. However, the biggest shortcoming of the neural network control algorithm is its black box nature, and the reasoning process and reasoning basis cannot be given for the output result. Furthermore, the application of the NNC relies on a large number of data training samples for learning and memory, and the convergence speed is slow. For the high-speed elevator car system, it is necessary to know the specific principle of vibration reduction, so as to guide the development of an active shock absorber for the car system. Meanwhile, considering the timeliness of shock absorber action and the randomness of external excitation during vibration reduction, it is impossible to obtain a large number of data to train the neural network. Therefore, neural network control algorithms need to be improved to have specific reasoning capabilities and the ability to use existing expert knowledge. Fuzzy control [33-35] is intelligent control based on the fuzzy set theory, fuzzy linguistic variables, and fuzzy logic reasoning. It has the ability to apply existing expert knowledge and experience and human language logic reasoning, along with the experience of successful control using fuzzy inference rules. It can carry out fuzzy reasoning on an uncertain system and can realize the intelligent control of a complex uncertain system. 


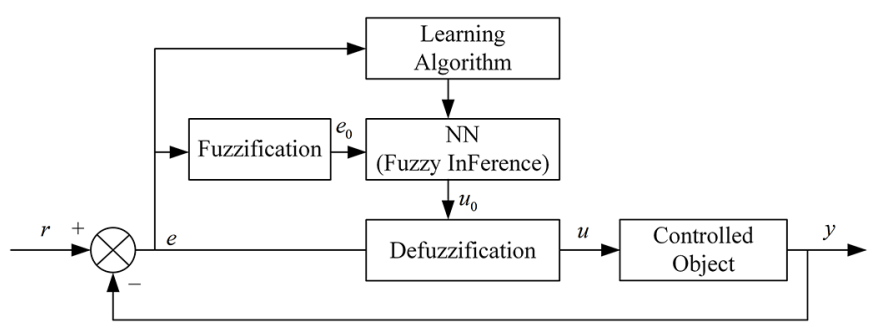

Fig. 14. Schematic of fuzzy neural network intelligent control system for the high-speed elevator based on the Mamdani model.
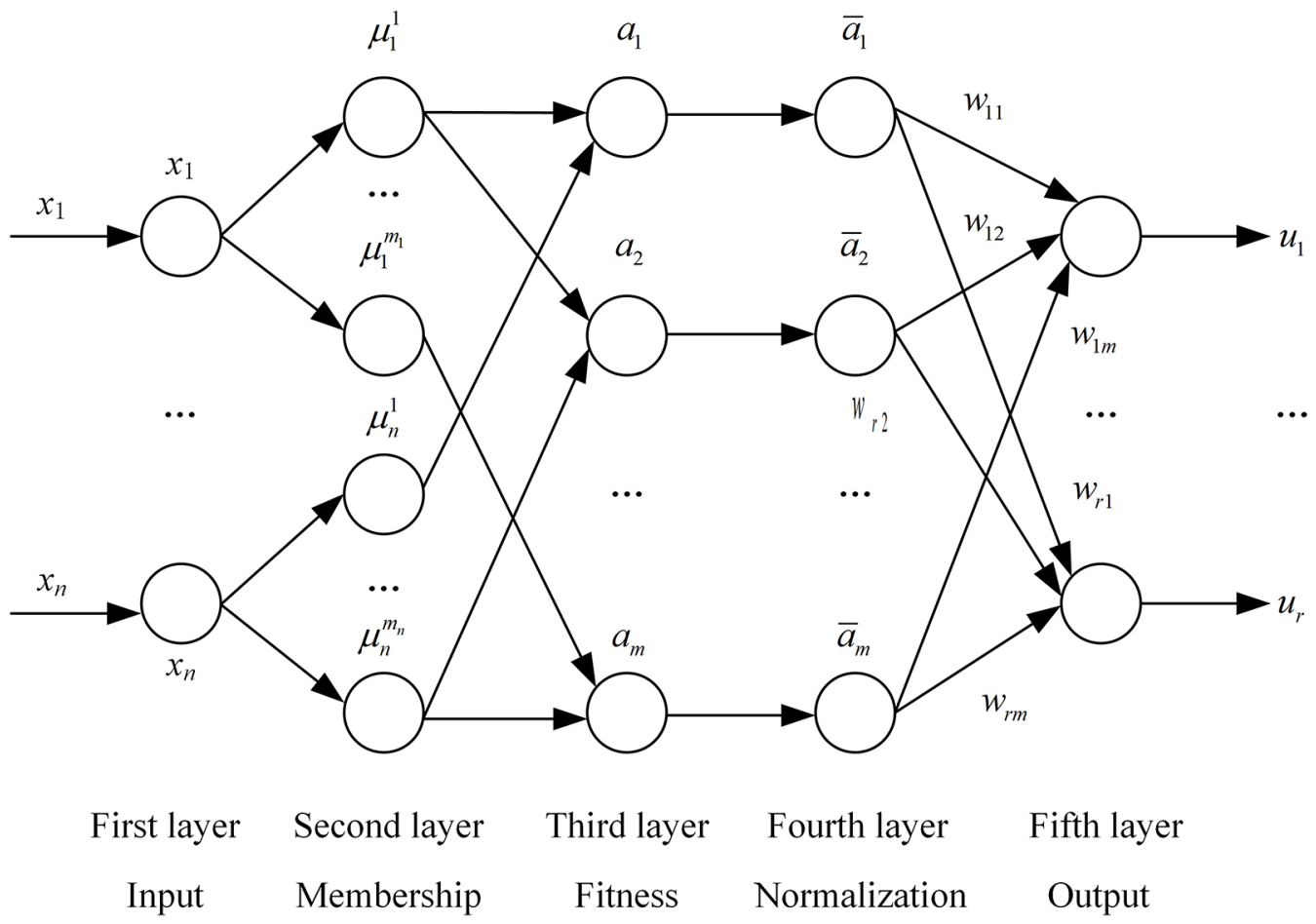

Fig. 15. Fuzzy neural network structure diagram based on the Mamdani model.

Therefore, in order to solve the problems of no learning, no clear reasoning basis and slow convergence speed in the existing high-speed elevator horizontal vibration control algorithms, the reasoning rules and logical reasoning of fuzzy control and the self-learning and memory of neural networks are combined in this study. The error between the actual output and the expected output of the car system are taken as inputs, and the fuzzy neural network intelligent vibration absorption controller based on the Mamdani model is designed. The control principle is shown in Figure 14.

The fuzzy neural network structure based on the Mamdani model [36,37] is shown in Figure 15. In the network, since the fuzzy segmentation number of each input component is predetermined, and the second layer membership function $\mu_{i}^{j}$ selects the bell-type function represented by the Gaussian function, it is only necessary to learn three parameters: the connection weight $w_{i j}$ of the fifth layer, and the central value $c_{i j}$ and the width value $\sigma_{i j}$ of the second layer membership function. Since the fuzzy neural network is essentially a multi-layer feed-forward network, the design of the learning algorithm is based on the error back propagation method of the BP neural network. The error of the actual output and the expected output are back-propagated in the five-layer network, thereby correcting the parameters $w_{i j}, c_{i j}$, and $\sigma_{i j}$.

The specific learning algorithm is as follows:

- Node function for each layer

First layer:

$$
\left\{\begin{array}{l}
\text { Input }: f_{i}^{(1)}=x_{i}^{(0)}=x_{i} \\
\text { Output }: x_{i}^{(1)}=g_{i}^{(1)}=f_{i}^{(1)}
\end{array},(i=1,2, \cdots, n)\right.
$$

Second layer:

$$
\left\{\begin{array}{l}
\text { Input }: f_{i j}^{(2)}=-\frac{\left(x_{i}^{(1)}-c_{i j}\right)^{2}}{\sigma_{i j}^{2}},(i=1,2, \cdots, n, j=1,2, \cdots, m) \\
\text { Output }: x_{i j}^{(2)}=\mu_{i}^{j}=g_{i j}^{(2)}=e^{f_{i j}^{(2)}}
\end{array}\right.
$$


Here, $c_{i j}$ and $\sigma_{i j}$ are the center and width values of the membership function $\mu_{i}^{j}$, respectively.

Third layer:

$$
\text { See equation (7) below. }
$$

Forth layer:

$$
\left\{\begin{array}{l}
\text { Input : } f_{j}^{(4)}=x_{j}^{(3)} / \sum_{i=1}^{m} x_{i}^{(3)}=\alpha_{j} / \sum_{i=1}^{m} \alpha_{i},(j=1,2, \cdots, m) . \\
\text { Output }: x_{j}^{(4)}=\bar{\alpha}_{j}=g_{j}^{(4)}=f_{j}^{(4)}
\end{array}\right.
$$

Fifth layer:

$$
\left\{\begin{array}{l}
\text { Input }: f_{i}^{(5)}=\sum_{j=1}^{m} w_{i j} x_{j}^{(4)}=\sum_{j=1}^{m} w_{i j} \bar{\alpha}_{j},(i=1,2, \cdots, r) \\
\text { Output }: x_{j}^{(5)}=y_{i}=g_{i}^{(5)}=f_{i}^{(5)}
\end{array}\right.
$$

- Error cost function

The error cost function is

$$
E=\frac{1}{2} \sum_{i=1}^{r}\left(t_{i}-y_{i}\right)^{2}
$$

where $t_{i}$ is the expected output, and $y_{i}$ is the actual output. - Parameter adjustment

The error back propagation algorithm is used to calculate $\frac{\partial E}{\partial w_{i j}}, \frac{\partial E}{\partial w_{i j}}$, and $\frac{\partial E}{\partial w_{i j}}$. The first-order gradient optimization algorithm is used to adjust uncertain parameters $w_{i j}, c_{i j}$, and $\sigma_{i j}$. The calculation process is as follows:

See equation (11) below.
Since $f^{(3)}$ uses multiplication, when $g_{i j}^{(2)}=\mu_{i}^{j}$ is an input to the rule point $k$, there are,

$$
s_{i j}=\frac{\partial f_{k}^{(3)}}{\partial g_{i j}^{(2)}}=\frac{\partial f_{k}^{(3)}}{\partial \mu_{i}^{j}}=\prod_{\substack{j=1 \\ j \neq i}}^{n} \mu_{j}^{i_{j}}
$$

Otherwise,

$$
s_{i j}=\frac{\partial f_{k}^{(3)}}{\partial g_{i j}^{(2)}}=\frac{\partial f_{k}^{(3)}}{\partial \mu_{i}^{j}}=0
$$

Therefore, the first-order gradient obtained is

$$
\left\{\begin{array}{l}
\frac{\partial E}{\partial w_{i j}}=\frac{\partial E}{\partial f_{i}^{(5)}} \frac{\partial f_{i}^{(5)}}{\partial w_{i j}}=-\delta_{i}^{(5)} x_{j}^{(4)}=-\left(t_{i}-y_{i}\right) \bar{\alpha}_{j} \\
\frac{\partial E}{\partial c_{i j}}=\frac{\partial E}{\partial f_{i j}^{(2)}} \frac{\partial f_{i j}^{(2)}}{\partial c_{i j}}=-\delta_{i j}^{(2)} \frac{2\left(x_{i}-c_{i j}\right)}{\sigma_{i j}^{2}} \\
\frac{\partial E}{\partial \sigma_{i j}}=\frac{\partial E}{\partial f_{i j}^{(2)}} \frac{\partial f_{i j}^{(2)}}{\partial \sigma_{i j}}=-\delta_{i j}^{(2)} \frac{2\left(x_{i}-c_{i j}\right)^{2}}{\sigma_{i j}^{3}}
\end{array}\right.
$$

Thus, according to equation (14), the learning algorithm with adjustable parameters can be obtained as follows:

$$
\left\{\begin{array}{l}
w_{i j}(k+1)=w_{i j}(k)-\beta \frac{\partial E}{\partial w_{i j}} \\
c_{i j}(k+1)=c_{i j}(k)-\beta \frac{\partial E}{\partial c_{i j}} \\
\sigma_{i j}(k+1)=\sigma_{i j}(k)-\beta \frac{\partial E}{\partial \sigma_{i j}}
\end{array}\right.
$$

where $\beta>0$ is the learning rate.

$$
\left\{\begin{array}{l}
\text { Input }: f_{j}^{(3)}=x_{1 i_{1}}^{(2)} x_{2 i_{2}}^{(2)} \cdots x_{n i_{n}}^{(2)}=\mu_{1}^{i_{1}} \mu_{2}^{i_{2}} \cdots \mu_{n}^{i_{n}} \\
\text { Output }: x_{j}^{(3)}=\alpha_{j}=g_{j}^{(3)}=f_{j}^{(3)}
\end{array},\left(j=1,2, \cdots, m, m=\prod_{i=1}^{n} m_{i}\right)\right.
$$

$$
\left\{\begin{array}{l}
\delta_{i}^{(5)}=-\frac{\partial E}{\partial f_{i}^{(5)}}=-\frac{\partial E}{\partial y_{i}}=t_{i}-y_{i} \\
\delta_{j}^{(4)}=-\frac{\partial E}{\partial f_{j}^{(4)}}=-\sum_{i=1}^{r} \frac{\partial E}{\partial f_{i}^{(5)}} \frac{\partial f_{i}^{(5)}}{\partial g_{j}^{(4)}} \frac{\partial g_{j}^{(4)}}{\partial f_{j}^{(4)}}=\sum_{i=1}^{r} \delta_{i}^{(5)} w_{i j} \\
\delta_{j}^{(3)}=-\frac{\partial E}{\partial f_{j}^{(3)}}=-\sum_{i=1}^{r} \frac{\partial E}{\partial f_{j}^{(4)}} \frac{\partial f_{j}^{(4)}}{\partial g_{j}^{(3)}} \frac{\partial g_{j}^{(3)}}{\partial f_{j}^{(3)}}=\delta_{j}^{(4)} \sum_{\substack{i=1 \\
i \neq j}}^{m} x_{i}^{(3)} /\left(\sum_{i=1}^{m} x_{i}^{(3)}\right)^{2}=\delta_{j}^{(4)} \sum_{\substack{i=1 \\
i \neq j}}^{m} \alpha_{i} /\left(\sum_{i=1}^{m} \alpha_{i}\right)^{2} \\
\delta_{i j}^{(2)}=-\frac{\partial E}{\partial f_{i j}^{(2)}}=-\sum_{k=1}^{m} \frac{\partial E}{\partial f_{k}^{(3)}} \frac{\partial f_{k}^{(3)}}{\partial g_{i j}^{(2)}} \frac{\partial g_{i j}^{(2)}}{\partial f_{i j}^{(2)}}=\sum_{k=1}^{m} \delta_{k}^{(3)} s_{i j} e^{f_{i j}^{(2)}}
\end{array}\right.
$$



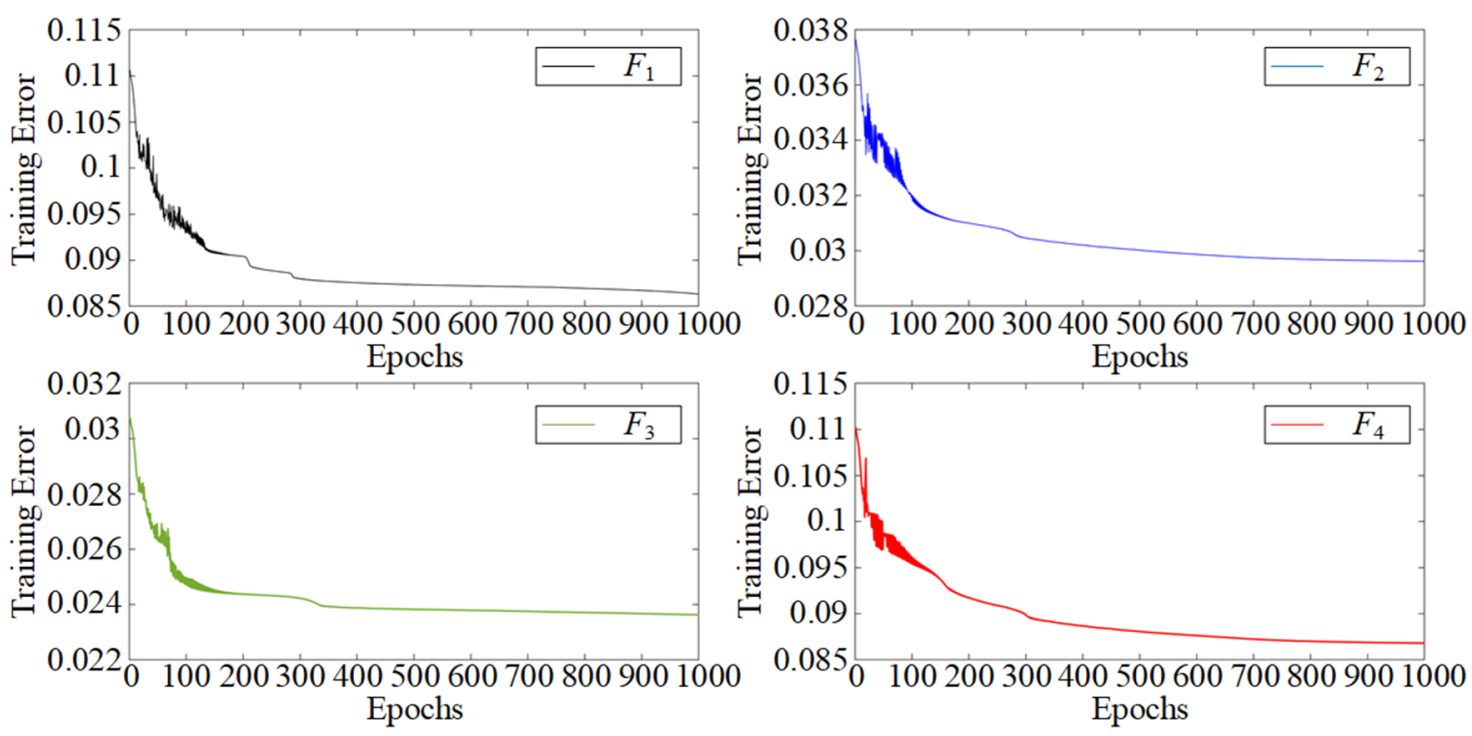

Fig. 16. Error of training.

\section{Simulation analysis}

This section takes a $4 \mathrm{~m} / \mathrm{s}$ high-speed elevator as an example to verify the feasibility and validity of the proposed intelligent control algorithm for the gas-liquid active guide shoes. Some simulation elevator parameters are shown in Table 1.

The fuzzy neural network control algorithm based on the Mamdani model is used to control the horizontal vibration acceleration at the bottom center position of the car and the car frame. In the algorithm, $e_{c}$ and $e_{f}$ are the input ( $e_{c}$ is the error between the actual horizontal vibration acceleration of the car and the expected value, $e_{f}$ is the error between the actual horizontal vibration acceleration of the car and the expected value), the control force $f_{i}(\mathrm{I}=1,2,3,4)$ is the output. $E_{c}, E_{f}$ and $f_{i}$ $(i=1,2,3,4)$ are domain values of $e_{c}, e_{f}$ and $f_{i}(i=1,2,3,4)$, all of which are $[0,1]$. The values of the fuzzy linguistic variables are negative big (NB), negative medium (NM), negative small (NS), zero (ZO), positive small (PS), positive medium (PM), and positive big (PB). The membership function uses a bell-type function represented by a Gaussian function, and the learning rate is $\beta=0.38$. The initial weights $w_{i j}(i=1,2,3,4$; $j=1,2, \ldots, 49)$ are random numbers on the interval $[0,1]$. The center value and width of the initial membership function are taken as random numbers in the interval [1, 1.6]. Therefore, the designed fuzzy neural network structure is 2-14-49-49-4.

\subsection{Algorithm training}

In order to ensure the effectiveness of the fuzzy neural network algorithm designed in this paper, it is necessary to train it and verify its convergence. Based on this, judge the accuracy of its output.
The input and output of training data are as follows:

$$
\left\{\begin{array}{l}
\text { Input }: E_{c}, E_{f} \\
\text { Output }: F_{i}(i=1,2,3,4)
\end{array}\right.
$$

In order to analyze the convergence of the algorithm, 300 groups of training data are randomly selected and 1000 epochs of training are used to train the designed fuzzy neural network. The training error of the proposed algorithm is shown in the Figure 16.

It can be seen from Figure 16 that in the fuzzy neural network designed in this paper, after about 500 steps of training, all four outputs converge, and the convergence error is less than 0.09 , so it can be seen that the fuzzy neural network control method proposed in this paper is convergent. On this basis, 100 groups of prediction outputs are made for the trained fuzzy neural network, and the result comparison figures of prediction output and expected output is obtained, as shown in Figure 17. The error between the predicted value and the expected value is shown in Figure 18. The average value and RMS values of the error between predicted and expected values of the four outputs are shown in Table 3 .

From Figures 17 to 18, it can be seen that the fitness between the predicted value of the trained fuzzy neural network and the expected value is high. Meanwhile, from Table 3, it can be seen that the average value and the RMS value of the errors between the predicted results and the expected results of the four outputs are less than 0.08 , that is to say, the predicted errors for the four output results are all within $10 \%$, so the prediction accuracy of the algorithm is high.

In conclusion, the convergence and prediction accuracy of the algorithm show that it is feasible to apply it to control the generation of the control force of the gas-liquid active guide shoe. 

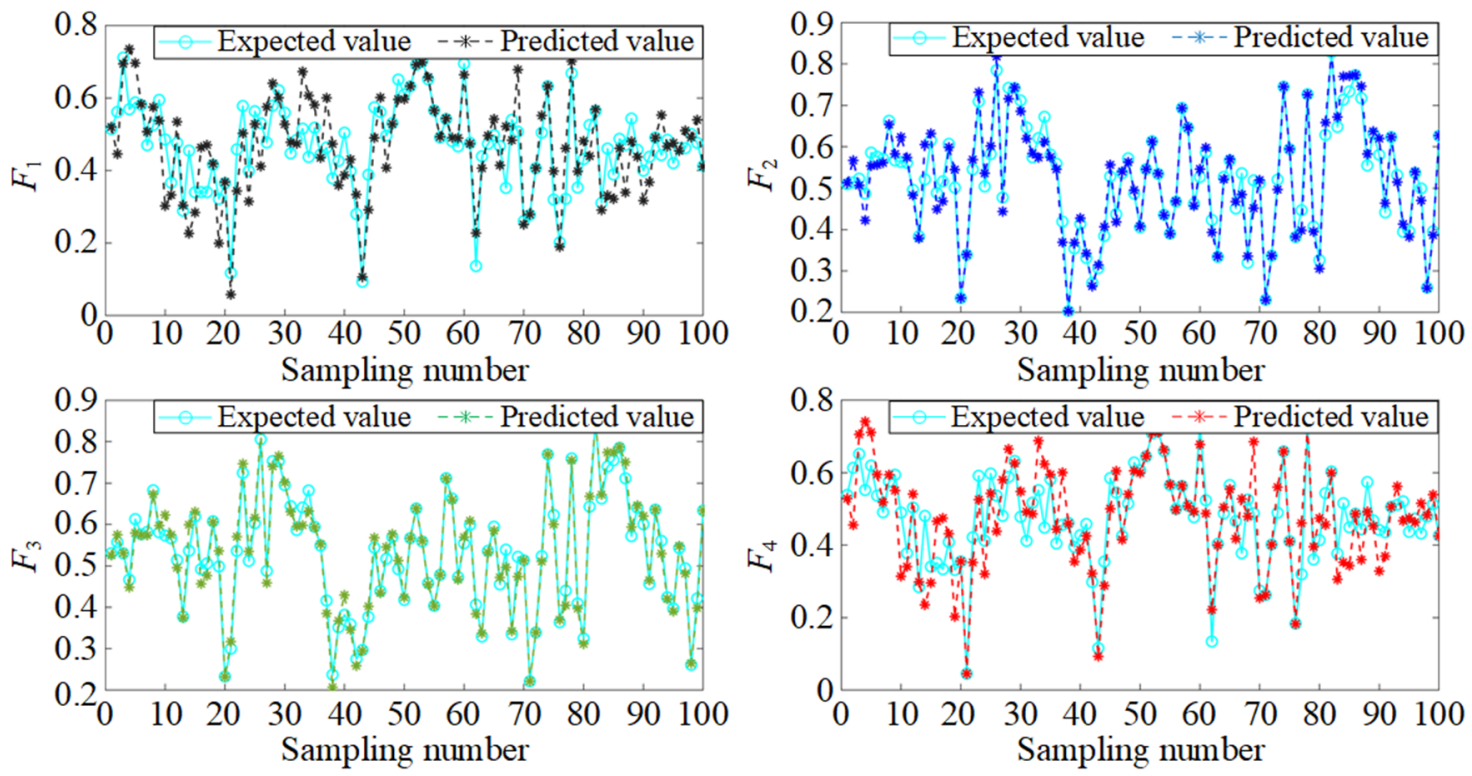

Fig. 17. Comparison between predicted value and expected value.
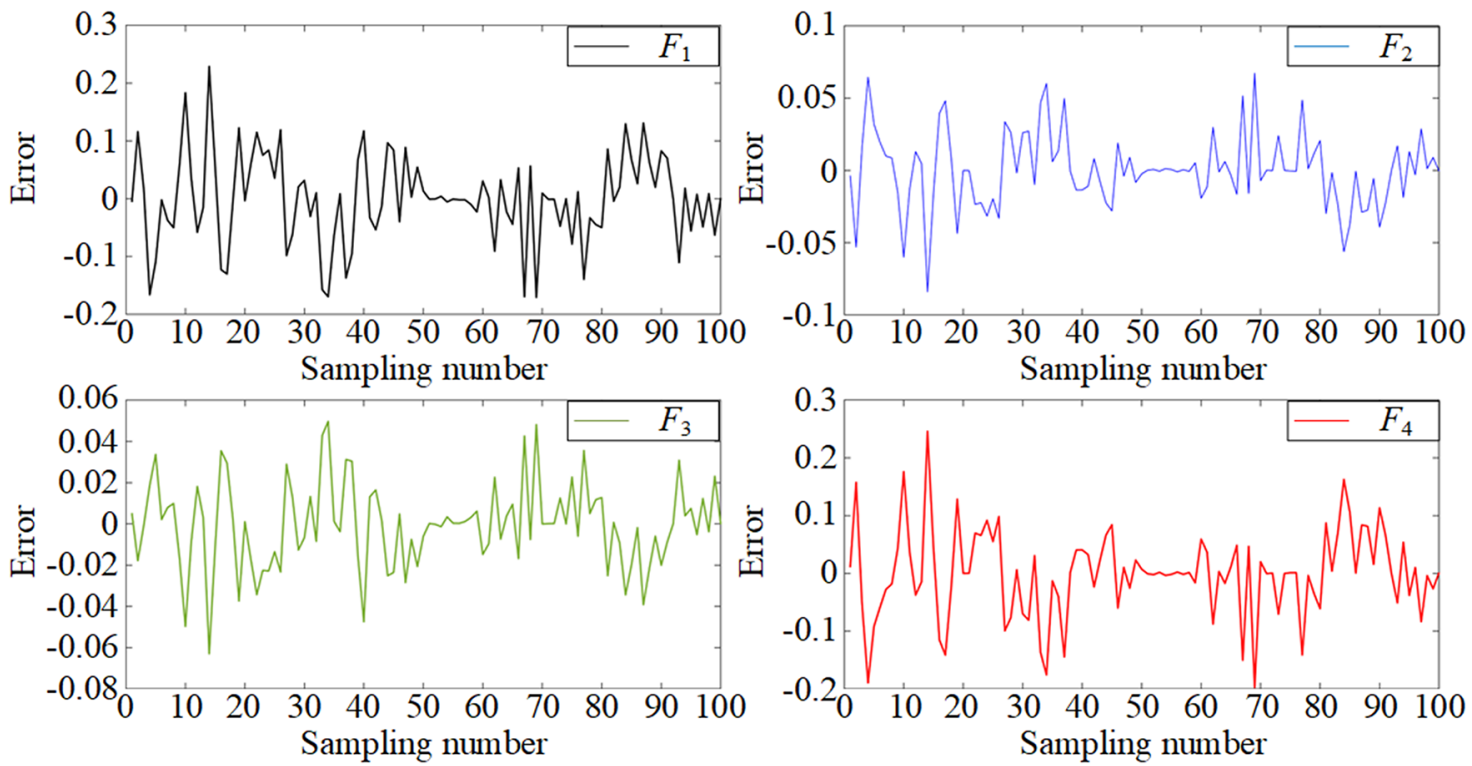

Fig. 18. Error between predicted value and expected value.

Table 3. Average value and RMS values of the error between predicted and expected values.

\begin{tabular}{lllll}
\hline & $F_{1}$ & $F_{2}$ & $F_{3}$ & $F_{4}$ \\
\hline Average value of errors & 0.0571 & 0.0184 & 0.0153 & 0.0539 \\
RMS value of errors & 0.0772 & 0.0261 & 0.0208 & 0.0764 \\
\hline
\end{tabular}

\subsection{Result analysis}

The state space model of the high-speed elevator car system is simulated by MATLAB, and the roughness excitation of the guide rail obeying the normal distribution is used as the interference input. Under three elevator operating conditions (no load, medium load, and full load), the timedomain and frequency-domain responses of the horizontal vibration acceleration of the car and the car frame are assessed under three control modes: uncontrolled, BP 




Fig. 19. Time-domain response of horizontal vibration acceleration of the elevator car with no load.

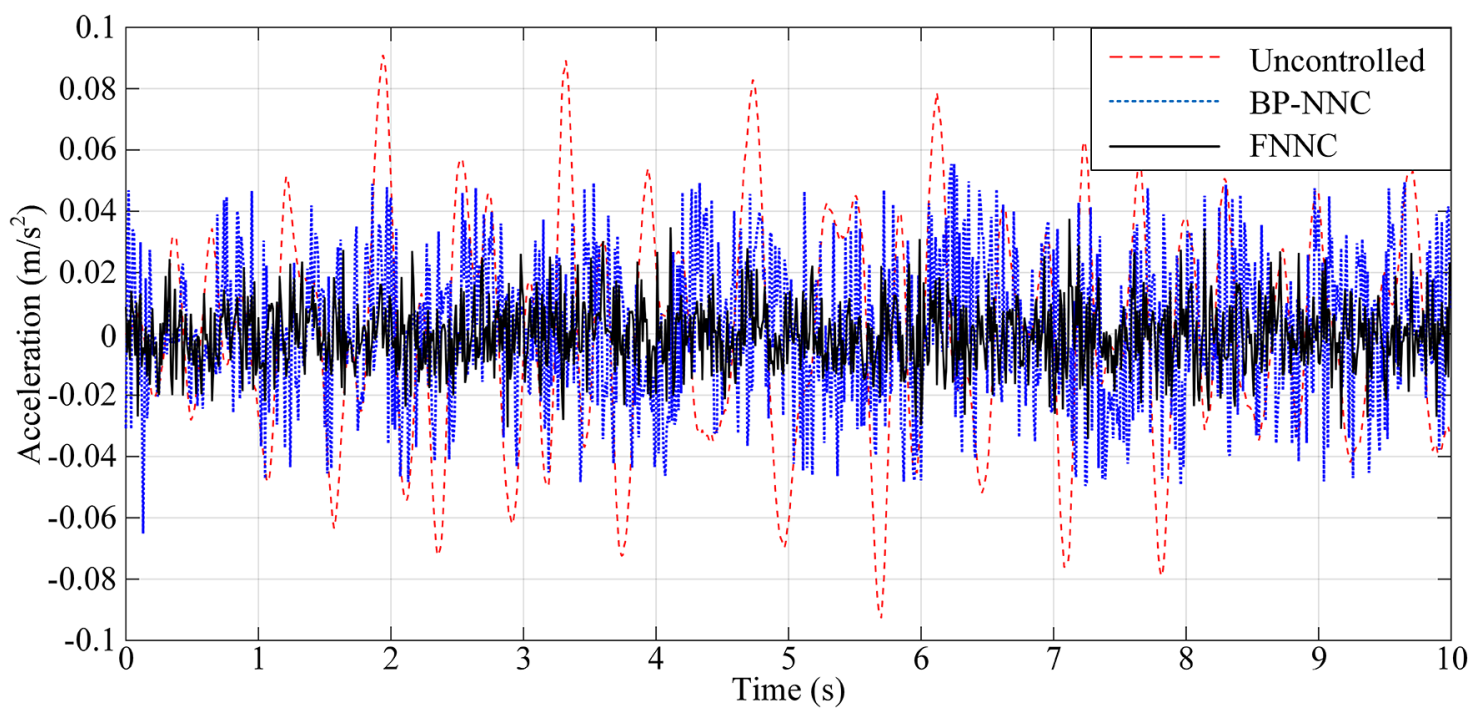

Fig. 20. Time-domain response of horizontal vibration acceleration of the elevator car with a medium load.

neural network control (BP-NNC), and fuzzy neural network control based on the Mamdani model (FNNC). These responses are compared and analyzed so as to verify the correctness and effectiveness of the control strategy designed in this study.

\subsubsection{Analysis results of horizontal vibration acceleration of the car}

In three operating conditions, the high-speed elevator uses three control modes: uncontrolled, BP-NNC, and FNNC. The time-domain response of the horizontal vibration acceleration is shown in Figures 19-21, and the frequencydomain response is shown in Figures 22-24.
In the time domain, it can be seen from Figures 19-21 that the BP-NNC and the FNNC can effectively reduce the horizontal vibration acceleration of the car under the three load conditions. However, at the beginning of the vibration, the vibration acceleration under the control of the BP-NNC is significantly larger than that under the uncontrolled mode. This is mainly due to the fact that the BP-NNC needs a lot of data training, and the BP-NNC has no specific reasoning basis and reasoning mode because of its black box nature and slow convergence speed; thus, the vibration suppression is poor at the beginning of the vibration. However, the FNNC has a fast convergence speed due to the inference rules of fuzzy control, and therefore, its overall vibration 


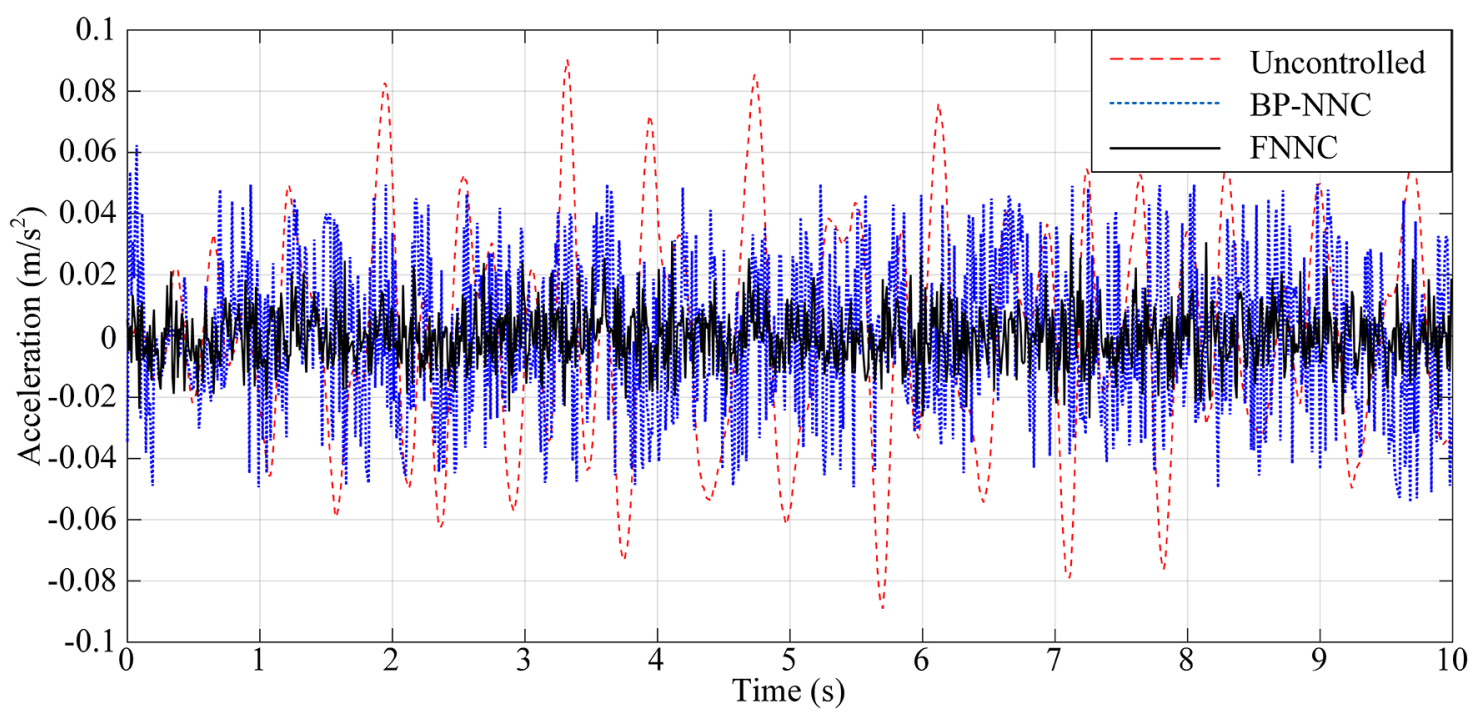

Fig. 21. Time-domain response of horizontal vibration acceleration of the elevator car with a full load.

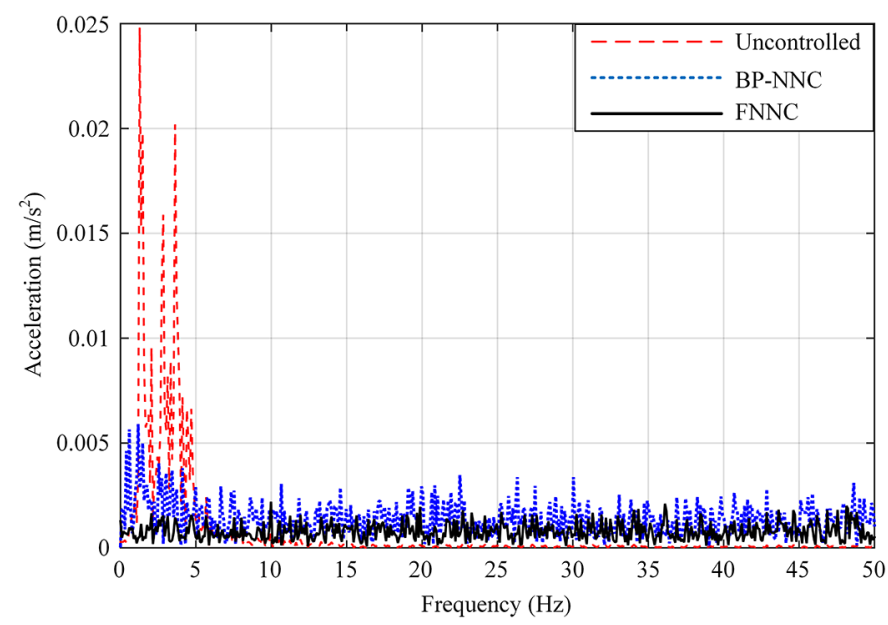

Fig. 22. Frequency-domain response of horizontal vibration acceleration of the elevator car with no load.

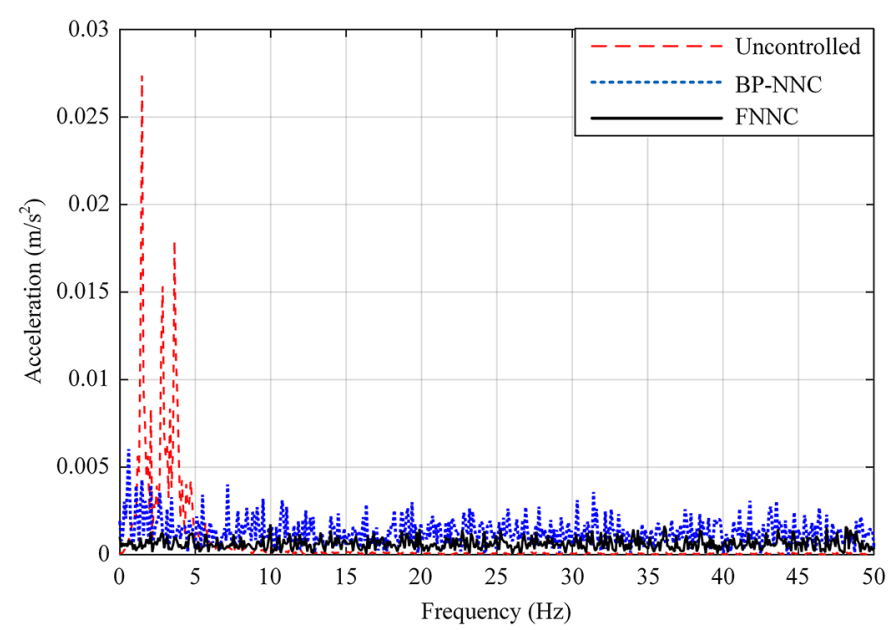

Fig. 23. Frequency-domain response of horizontal vibration acceleration of the elevator car with a medium load.

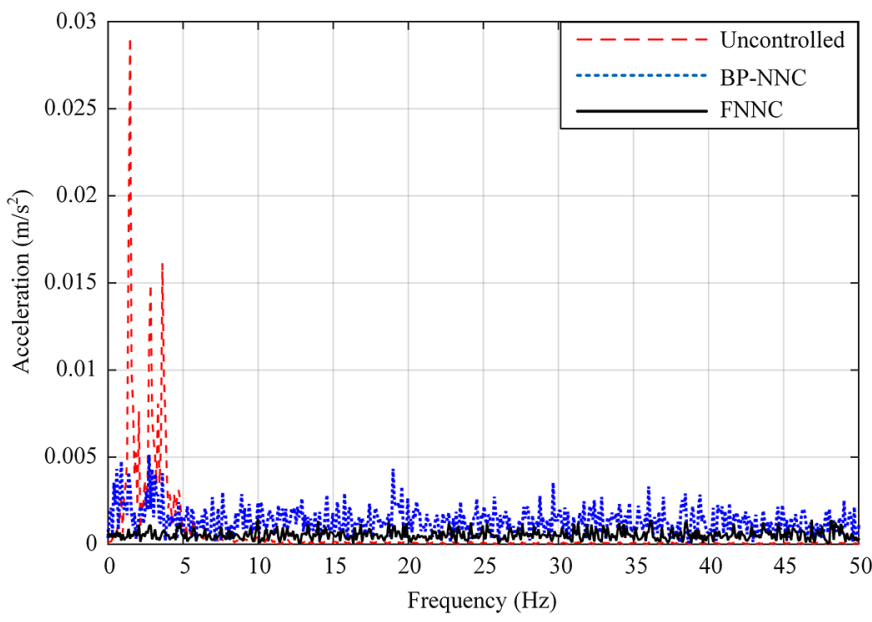

Fig. 24. Frequency-domain response of horizontal vibration acceleration of the elevator car with a full load.

reduction is superior; the horizontal vibration of the car can be effectively reduced during the whole vibration process.

Since the main evaluation indices of the high-speed elevator vibration reduction effect are the RMS, mean, and maximum values of the vibration acceleration, these values under the three load conditions are listed in Table 4. Under the no load condition, compared with using the uncontrolled mode, using the BP-NNC, the RMS, mean, and maximum values of the horizontal vibration acceleration of the car are reduced by $36.8 \%, 36.3 \%$, and $26.2 \%$, respectively. However, under the action of the FNNC, these values are reduced by $65.2 \%, 65.8 \%$, and $57.3 \%$, respectively. Under the medium load condition, compared with using uncontrolled mode, when using the BP-NNC, the RMS, mean, and maximum values of the horizontal vibration acceleration of the car are reduced by $33.6 \%$, $33.8 \%$, and $29.8 \%$, respectively. However, under the action of the FNNC, these values are reduced by $68.9 \%, 70.0 \%$, 
Table 4. Time-domain response results of horizontal vibration acceleration of the elevator car.

\begin{tabular}{|c|c|c|c|c|c|}
\hline & & & $\operatorname{RMS}\left(\mathrm{m} / \mathrm{s}^{2}\right)$ & $\operatorname{MEAN}\left(\mathrm{m} / \mathrm{s}^{2}\right)$ & $\operatorname{MAX}\left(\mathrm{m} / \mathrm{s}^{2}\right)$ \\
\hline \multirow{9}{*}{ Elevator car } & \multirow{3}{*}{ Uncontrolled } & No load & 0.0402 & 0.0325 & 0.1112 \\
\hline & & Medium load & 0.0357 & 0.0293 & 0.0927 \\
\hline & & Full load & 0.0357 & 0.0294 & 0.0903 \\
\hline & \multirow{4}{*}{ BP-NNC } & No load & 0.0254 & 0.0207 & 0.0821 \\
\hline & & Medium load & 0.0237 & 0.0194 & 0.0651 \\
\hline & & Full load & 0.0247 & 0.0205 & 0.0624 \\
\hline & & No load & 0.0140 & 0.0111 & 0.0475 \\
\hline & \multirow{2}{*}{ FNNC } & Medium load & 0.0111 & 0.0088 & 0.0375 \\
\hline & & Full load & 0.0097 & 0.0077 & 0.0331 \\
\hline
\end{tabular}

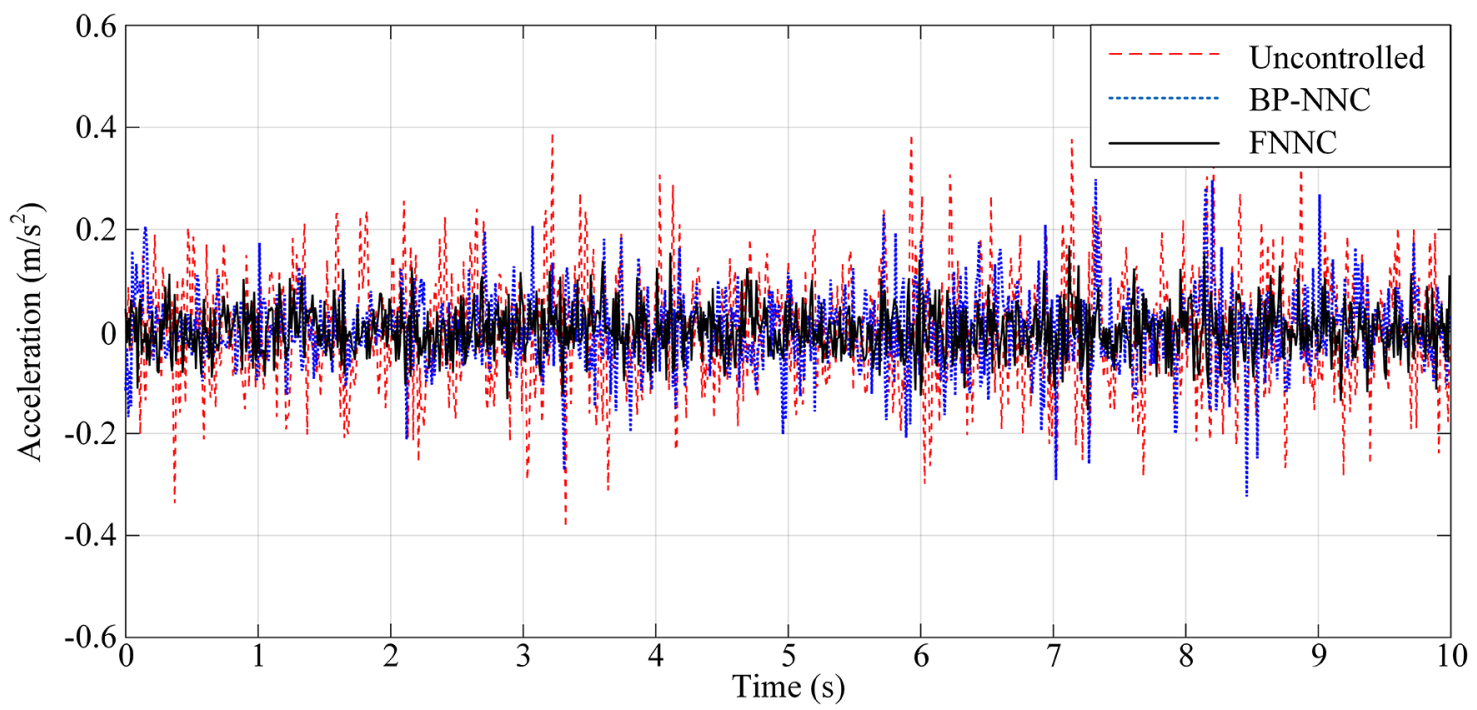

Fig. 25. Time-domain response of horizontal vibration acceleration of the elevator car frame with no load.

and $59.5 \%$, respectively. Under the full load condition, compared with using the uncontrolled mode, by using the BP-NNC, the RMS, mean, and maximum values of the horizontal vibration acceleration of the car are reduced by $30.8 \%, 30.3 \%$, and $30.9 \%$, respectively. However, under the action of the FNNC, these values are reduced by $72.8 \%$, $73.8 \%$, and $63.3 \%$ respectively. From comparing the acceleration evaluation indices under the three load conditions, we see that the FNNC has a good suppression effect on the horizontal vibration of the car.

In the frequency domain, it can be seen from Figures 22-24 that the BP-NNC and the FNNC have significant effects on the reduction of the vibration amplitude of the car under the three operating conditions. Furthermore, the trend of the car vibration frequency distribution curve does not change when the vibration amplitude of the car is reduced. Meanwhile, it can be seen from Figures 22-24 that the FNNC is superior to the BP neural network control method when reducing the vibration amplitude. Therefore, in the frequency domain, the FNNC has an effective inhibitory effect on the horizontal vibration of the car, and the suppression effect is superior to that of the BP-NNC.
The analysis results of the horizontal vibration acceleration of the integrated car in the time domain and the frequency domain show that the FNNC can effectively reduce the horizontal vibration of the car. The results also show that the FNNC's suppression effect is better than that of the BP-NNC.

\subsubsection{Analysis results of horizontal vibration acceleration of the car frame}

The time-domain response of the horizontal vibration acceleration of the car frame under three operating conditions and three control modes (uncontrolled, BPNNC, and FNNC) are shown in Figures 25-27. The frequency-domain responses are shown in Figures 28-30.

In the time domain, it can be seen from Figures 25-27 that under the three operating conditions of the elevator, the BP-NNC and the FNNC have a good suppression effect on the horizontal vibration of the car frame. However, at the beginning of the vibration, the vibration acceleration of the car frame under the BP-NNC is larger than the vibration acceleration under the uncontrolled effect. The reason for this phenomenon is the same as that explained in Section 5.1, and 


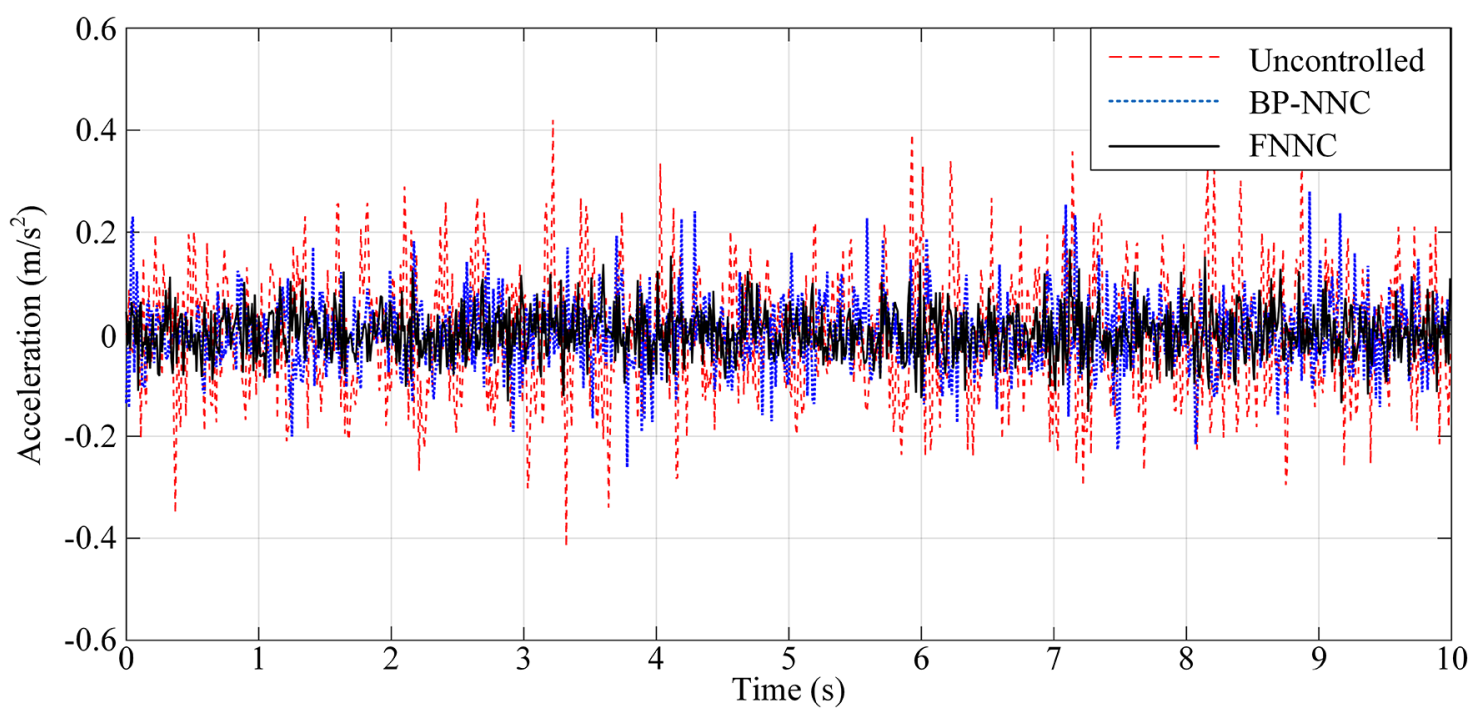

Fig. 26. Time-domain response of horizontal vibration acceleration of the elevator car frame with a medium load.

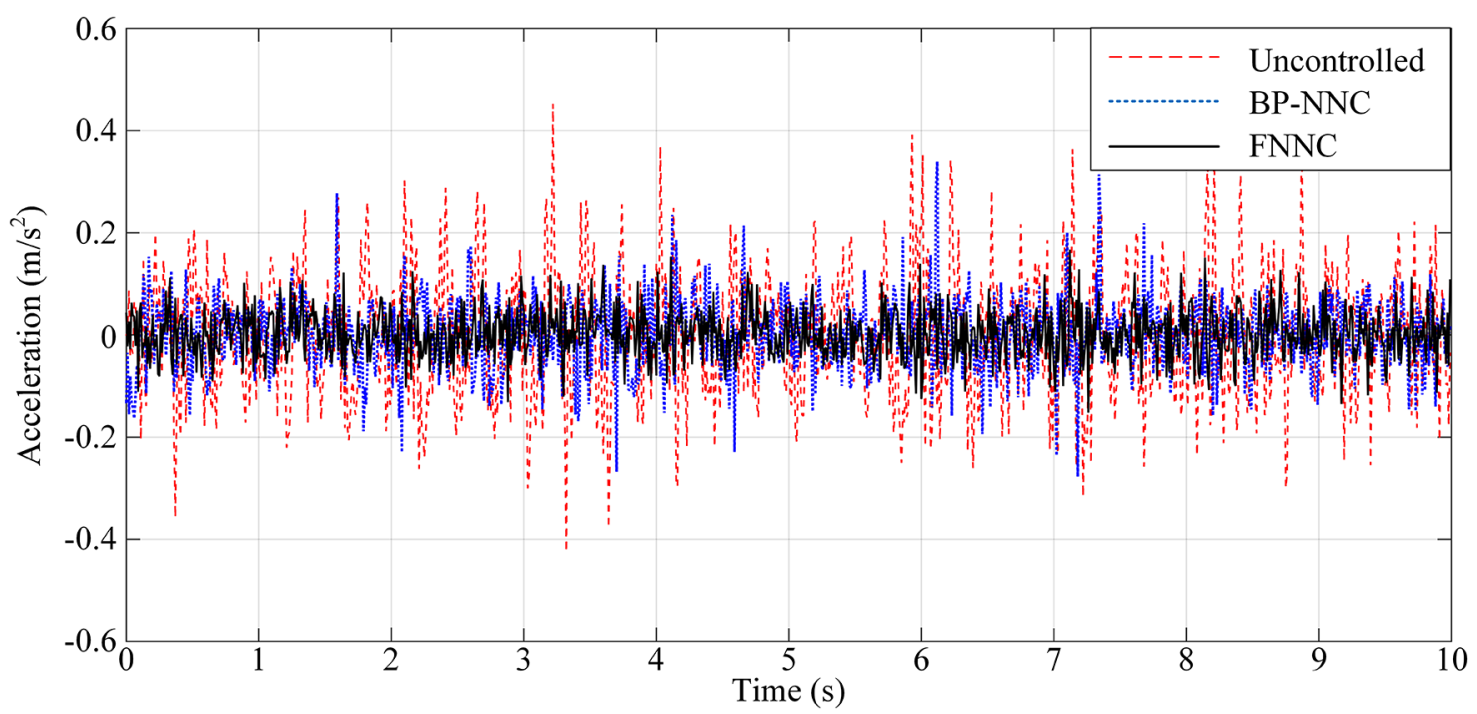

Fig. 27. Time-domain response of horizontal vibration acceleration of the elevator car frame with a full load.

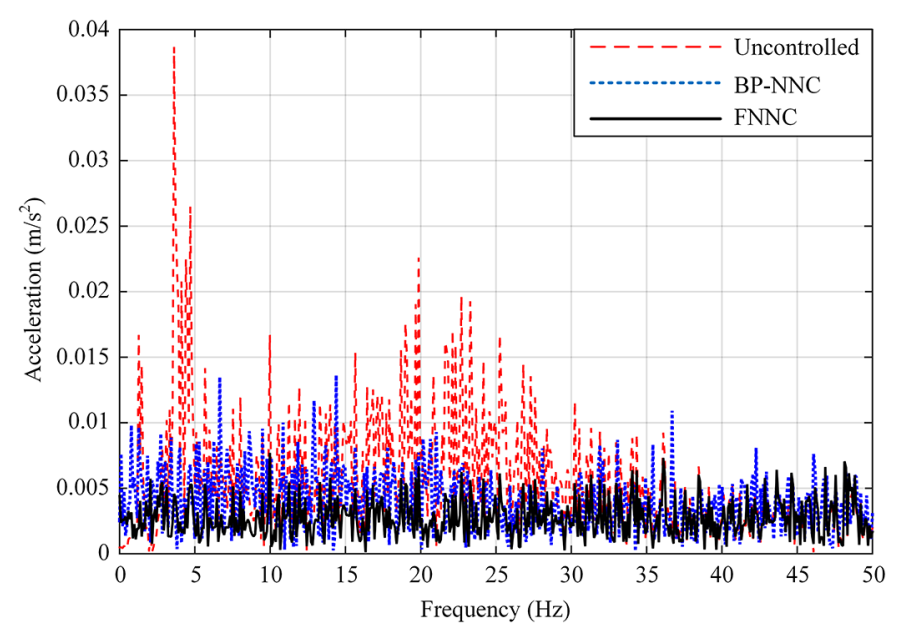

Fig. 28. Frequency-domain response of horizontal vibration acceleration of the elevator car frame with no load.

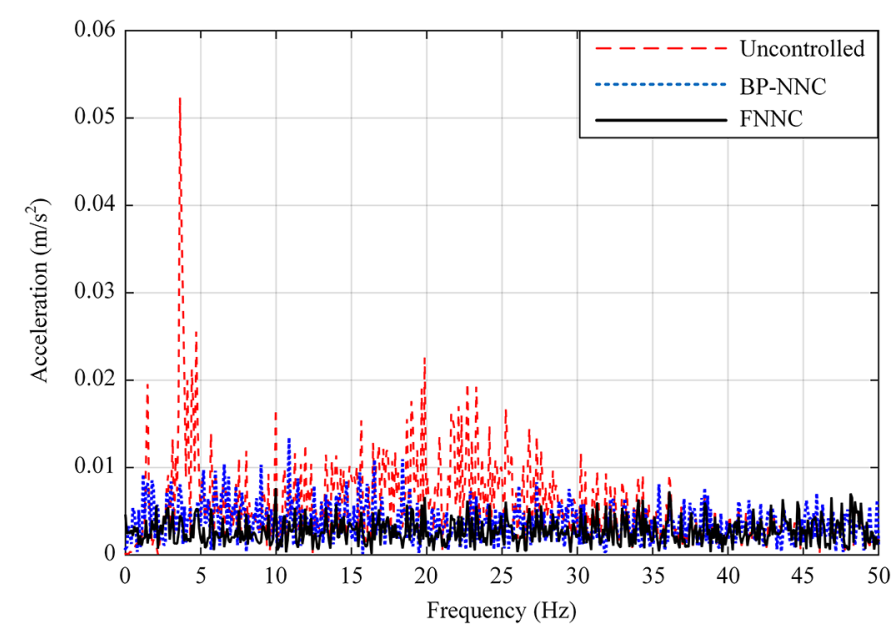

Fig. 29. Frequency-domain response of horizontal vibration acceleration of the elevator car frame with a medium load. 
Table 5. Time-domain response results of horizontal vibration acceleration of the elevator car frame.

\begin{tabular}{|c|c|c|c|c|c|}
\hline & & & $\operatorname{RMS}\left(\mathrm{m} / \mathrm{s}^{2}\right)$ & $\operatorname{MEAN}\left(\mathrm{m} / \mathrm{s}^{2}\right)$ & $\operatorname{MAX}\left(\mathrm{m} / \mathrm{s}^{2}\right)$ \\
\hline \multirow{9}{*}{ Elevator car frame } & \multirow{3}{*}{ Uncontrolled } & No load & 0.1144 & 0.0914 & 0.3905 \\
\hline & & Medium load & 0.1197 & 0.0955 & 0.4202 \\
\hline & & Full load & 0.1241 & 0.0992 & 0.4525 \\
\hline & \multirow{4}{*}{ BP-NNC } & No load & 0.0716 & 0.0531 & 0.3234 \\
\hline & & Medium load & 0.0670 & 0.0507 & 0.2799 \\
\hline & & Full load & 0.0702 & 0.0536 & 0.3395 \\
\hline & & No load & 0.0491 & 0.0389 & 0.1686 \\
\hline & \multirow{2}{*}{ FNNC } & Medium load & 0.0489 & 0.0387 & 0.1671 \\
\hline & & Full load & 0.0488 & 0.0386 & 0.1671 \\
\hline
\end{tabular}

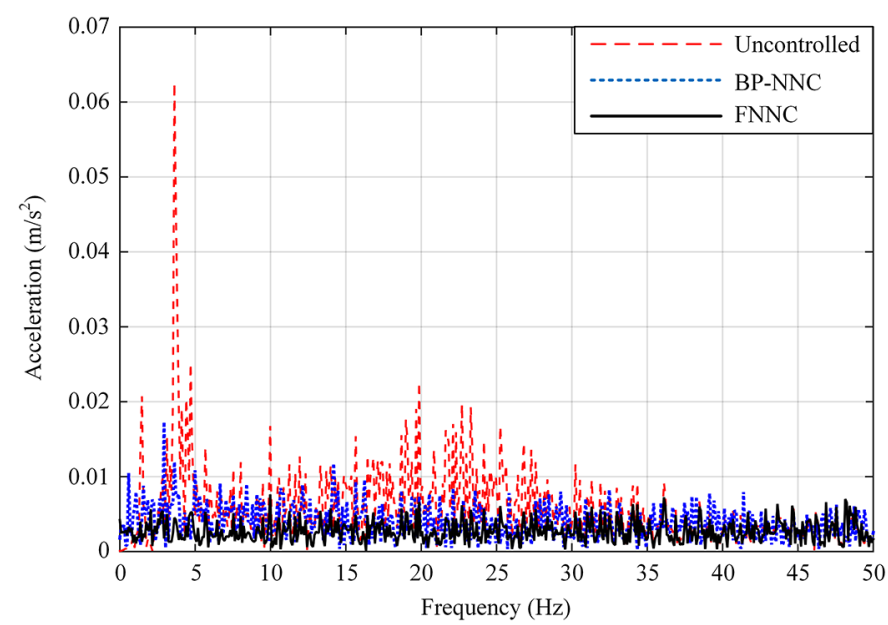

Fig. 30. Frequency-domain response of horizontal vibration acceleration of the elevator car frame with a full load.

will not be repeated here. It can be seen from the graph that the suppression effect of the FNNC is better than that of the BP-NNC.

Here, we adopt the same analysis method as that employed in Section 5.1. The RMS, mean, and maximum values of the horizontal vibration acceleration of the car frame under the three load conditions are shown in Table 5. Under the no load condition, compared with using the uncontrolled mode, by using the BP-NNC, the RMS, mean, and maximum values of the horizontal vibration acceleration of the car frame are reduced by $37.4 \%, 41.9 \%$, and $17.2 \%$, respectively. However, under the action of the FNNC, these values are reduced by $57.1 \%, 57.4 \%$, and $56.8 \%$, respectively. Under the medium load condition, compared with using the uncontrolled mode, when using the BP-NNC, the RMS, mean, and maximum values of the horizontal vibration acceleration of the car frame are reduced by $44.0 \%, 46.9 \%$, and $33.4 \%$, respectively. However, under the action of the FNNC, these values are reduced by $59.1 \%, 59.5 \%$, and $60.2 \%$, respectively. Under the full load condition, compared with using the uncontrolled mode, by using the BP-NNC, the RMS, mean, and maximum values of the horizontal vibration acceleration of the car frame are reduced by $43.4 \%, 46.0 \%$, and
$25.0 \%$, respectively. However, under the action of the FNNC, these values are reduced by $60.7 \%, 61.1 \%$, and $63.1 \%$, respectively. By comparing the horizontal vibration acceleration data of the car frame under three load conditions, it can be concluded that the FNNC can effectively reduce the horizontal vibration of the car frame in the time domain. Moreover, the FNNC's suppression effect is better than that of the BP-NNC.

It can be seen from Figures 28-30 that the BP-NNC and FNNC effectively reduce the vibration amplitude of the car frame in the frequency domain under the three operating conditions. Furthermore, the trend of the vibration frequency distribution curve of the car frame does not change when the vibration amplitude of the car frame is reduced. Meanwhile, we can see that the FNNC reduces the vibration amplitude of the car frame more than the BP-NNC. Therefore, in the frequency domain, it is concluded that the FNNC has an effective suppression effect on the horizontal vibration of the car frame, and this suppression effect is better than that of the BP-NNC.

The analysis results of the horizontal vibration acceleration in the time and frequency domains show that the FNNC designed in this study can suppress the horizontal vibration of the car frame. Furthermore, its suppression effect is better than that of the BP-NNC.

\section{Conclusion}

- In this study, a gas-liquid active guide shoe for the highspeed elevator is designed using a gas-liquid cylinder and a gas-liquid converter. This design overcomes the shortcomings of oil leakage and slow operation speed of hydraulic active guide shoes, and provides a new scheme for the active vibration reduction of horizontal vibration of high-speed elevators.

- A horizontal vibration model of 8-DOF high-speed elevator car system with separated car and car frame is established. The dynamic equation and state space equation of car system are obtained. Then the simulation results and the measured results are analyzed in time and frequency domains. This verifies the correctness of the model and provides a model basis for the research of active vibration control of high-speed elevators. 
- By combining fuzzy control with neural network control, a fuzzy neural network intelligent control algorithm based on the Mamdani model is designed. The algorithm has the advantages of the fuzzy control algorithm (such as good robustness and strong logical reasoning ability) and has the intelligent processing ability of neural networks. The proposed model is suitable for active vibration reduction of the high-speed elevator car system.

- Through MATLAB simulation analysis, under three operating conditions (no load, medium load, and full load), the time-domain and the frequency-domain responses of the horizontal vibration acceleration of the bottom center position of the car and the car frame under three control methods (uncontrolled, BP neural network control, and fuzzy neural network control) are compared and analyzed. The results show that under the three load conditions, compared with using the uncontrolled mode, the fuzzy neural network control algorithm designed in this study decreases the RMS, mean, and maximum values of the horizontal vibration acceleration of the car and the car frame by more than $55 \%$. Furthermore, the effect of vibration reduction is better than that of the BP neural network control. The results verify the feasibility and effectiveness of the proposed control method.

This study was funded by the Shandong Province Natural Science Foundation, China (grant number ZR2017MEE049) and the Key Research Development Project of Shandong Province, China (grant number 2018GSF121004). We also thank LetPub (www. letpub.com) for its linguistic assistance during the preparation of this manuscript. The authors sincerely thank the editors and reviewers for their insights and comments to further improve the quality of this manuscript.

\section{References}

[1] W.D. Zhu, Y. Chen, Forced response of translating media with variable length and tension: application to high-speed elevators, Proceedings of the Institution of Mechanical Engineers Part K Journal of Multi-body Dynamics 219, $35-53(2004)$

[2] R. Monge, J.M. Rodríguez-Fortún, A. Gómez, J.A. Roig, P. González, Design of semi-active roller guides for high speed elevators, Applied Mechanics and Materials 706, 108-116 (2015)

[3] B.H. Xia, X. Shi, Horizontal vibrations of high-speed elevator with guide rail excitation, Machine Building \& Automation 41, 161-165 (2012)

[4] R. Tao, Y.C. Xu, F.H. Deng, S. Guo, H. Wang, Feature extraction of an elevator guide shoe vibration signal based on SVD optimizing LMD, Journal of Vibration and Shock 36, 166-171 (2017)

[5] Y.H. Feng, J.W. Zhang, Hydraulic active guide roller system for high-speed elevator based on fuzzy controller, Chin. Journal of Mechanical Engineering. 20, 68-73 (2007)
[6] J.H. Bao, P. Zhang, C.M. Zhu, Vibration control of highspeed elevator hoisting systems based on tensioning devices, Journal of Vibration and Shock 36, 221-226 (2017)

[7] Y.T. Ai, Z. Wang, L.X. Li, Research on active vibration control technology of high-speed traction elevator, Vibration and Shock 26, 56-58 (2007)

[8] Y.H. Feng, J.W. Zhang, The modeling and simulation of horizontal vibrations for high-speed elevator, Journal of Shanghai Jiaotong University 41, 557-560 (2007)

[9] Y.H. Feng, J.W. Zhang, X. Zhang, P. Sun, Study of active control of horizontal vibrations for high-speed elevator, Mechanical Science and Technology for Aerospace Engineering 26, 1076-1079 (2007)

[10] Y.H. Feng, J.W. Zhang, Study on active control strategy of horizontal vibrations of high-speed elevator, Journal of System Simulation 19, 843-845 (2007)

[11] Y.H. Feng, J.W. Zhang, Y.E. Zhao, Modeling and robust control of horizontal vibrations for high-speed elevator, Journal of Vibration and Control 15, 1375-139 (2009)

[12] L. Wang, Adaptive fuzzy control for hydraulic active guide shoe of high-speed elevator, Mechanical Design and Manufacturing 2011, 178-180 (2011)

[13] L. Wang, Y.H. Feng, J.L. Xue, Design of hydraulic active boot control system for high-speed elevator based on Aduc7026, Machinery Manufacturing 49, 25-29 (2011)

[14] K. Nakano, R. Hayashi, Y. Suda, N. Noguchi, A. Arakawa, Active vibration control of an elevator car using two rotary actuators, Journal of System Design and Dynamics 5, 155-163 (2011)

[15] N. Naoaki, A. Atsushi, K. Miyata, T. Yoshimura, S. Shin, Study on active vibration control for high-speed elevators, Journal of System Design and Dynamics 5, 164-179 (2011)

[16] J.L. Xue, Y.H. Feng, H.X. Wu, Study on active control of horizontal vibration for high-speed elevators based on generalized predictive PID, Mechanical Science and Technology for Aerospace Engineering 31, 1222-1226 (2012)

[17] D.R. Santo, J.M. Balthazar, A.M. Tusset, V. Piccirilo, R. Brasil, M. Silveira, On nonlinear horizontal dynamics and vibrations control for high-speed elevators, Journal of Vibration and Control 24, 825-838 (2018)

[18] Q. Zhang, Z. Yang, C. Wang, Y.H. Yang, R.J. Zhang, Intelligent control of active shock absorber for high-speed elevator car, Proceedings of the Institution of Mechanical Engineers, Part C: Journal of Mechanical Engineering Science 233, 3804-3815 (2018)

[19] S.X. Cao, Q. He, R.J. Zhang, Robust control of high-speed elevator transverse vibration based on LMI optimization, IOP Conference Series Materials Science and Engineering 538, 012032 (2019)

[20] R.J. Zhang, C. Wang, Q. Zhang, J. Liu, Response analysis of non-linear compound random vibration of a high-speed elevator, Journal of Mechanical Science and Technology 33, 51-63 (2019)

[21] R.J. Zhang, C. Wang, Q. Zhang, Response analysis of the composite random vibration of a high-speed elevator considering the nonlinearity of guide shoe, Journal of the Brazilian Society of Mechanical Sciences and Engineering 40, 90 (2018) 
[22] K. Utsunomiya, K.I. Okamoto, T. Yumura, K. Funai, H. Kuraoka, Active roller guide system for high-speed elevators, Elevator World 50, 86-92 (2002)

[23] H.L. Wang, Statistical error characteristic Analysis of Surface Roughness signal, Shanghai Metrology 43, 18-22 (2016)

[24] Z. Yang, Q. Zhang, R.J. Zhang, L.Z. Zhang, Transverse vibration response of a super high-speed elevator under air disturbance, International Journal of Structural Stability and Dynamics 19, 164-179 (2019)

[25] J. Liu, R.J. Zhang, Q. He, Q. Zhang, Study on horizontal vibration characteristics of high-speed elevator with airflow pressure disturbance and guiding system excitation, Mechanics \& Industry 20, 305 (2019)

[26] M.C. Zhou, J. Yan, J.W. Liu, Detection and Measurement, Chemical Industry Press, Beijing, 2004

[27] S. Qiao, R.J. Zhang, Q. He, L.Z. Zhang, Theoretical modeling and sensitivity analysis of the car-induced unsteady airflow in super high-speed elevator, Journal of Wind Engineering and Industrial Aerodynamics 188, 280-293 (2019)

[28] L.M. Qiu, Z.L. Wang, S.Y. Zhang, J. Chen, A vibrationrelated design parameter optimization method for highspeed elevator horizontal vibration reduction, Shock and Vibration 2020, 1-20 (2020)

[29] H.Q. Jia, Applied statistics, 5th Edition, University of international business and Economics Press, Beijing, 2010

[30] D. Lehký, O. Slowik, D. Novák, Reliability-based design: artificial neural networks and double-loop reliability-based optimization approaches, Advances in Engineering Software 117, 123-135 (2018)

[31] Z. Li, H. Adeli, Control methodologies for vibration control of smart civil and mechanical structures, Expert Syst. 35, e12354 (2018)

[32] D. Pan, F. Gao, Y. Miao, R. Cao, Co-simulation research of a novel exoskeleton-human robot system on humanoid gaits with fuzzy-PID/PID algorithms, Advances in Engineering Software 79, 36-46 (2015)

[33] G. Tairidis, G. Foutsitzi, P. Koutsianitis, G.E. Stavroulakis, Fine tuning of a fuzzy controller for vibration suppression of smart plates using genetic algorithms, Advances in Engineering Software 101, 123-135 (2016)

[34] H.J. Gao, W. He, Y.H. Song, S. Zhang, C.Y. Sun, Modeling and neural network control of a flexible beam with unknown spatiotemporally varying disturbance using assumed mode method, Neurocomputing 314, 458-467 (2018)

[35] V.B. Bui, Q.C. Tran, H.L. Bui, Multi-objective optimal design of fuzzy controller for structural vibration control using Hedge-algebras approach, Artificial Intelligence Review 50, 569-595 (2017)

[36] C.Y. Sun, H.J. Gao, W. He, Y. Yu, Fuzzy neural network control of a flexible robotic manipulator using assumed mode method, IEEE Transactions on Neural Networks and Learning Systems 29, 5214-5227 (2018)

[37] Z.C. Qiu, T.X. Wang, Fuzzy neural network vibration control on a piezoelectric flexible hinged plate using stereo vision detection, Journal of Intelligent Material Systems and Structures 30, 556-575 (2019)

Cite this article as: Q. He, P. Zhang, S. Cao, R. Zhang, Q. Zhang, Intelligent control of horizontal vibration of high-speed elevator based on gas-liquid active guide shoes, Mechanics \& Industry 22, 2 (2021) 